\title{
GEOMETRIC FLOW ON COMPACT LOCALLY CONFORMALLY KÄHLER MANIFOLDS
}

\author{
YOSHINOBU KAMISHIMA AND LIVIU ORNEA
}

\begin{abstract}
We study two kinds of transformation groups of a compact locally conformally Kähler (l.c.K.) manifold. First we study compact l.c.K. manifolds by means of the existence of holomorphic l.c.K. flow (i.e., a conformal, holomorphic flow with respect to the Hermitian metric.) We characterize the structure of the compact l.c.K. manifolds with parallel Lee form. Next, we introduce the Lee-Cauchy-Riemann (LCR) transformations as a class of diffeomorphisms preserving the specific $G$-structure of l.c.K. manifolds. We show that compact l.c.K. manifolds with parallel Lee form admitting a $\mathbb{C}^{*}$ flow of LCR transformations are rigid: it is holomorphically isometric to a Hopf manifold with parallel Lee form.
\end{abstract}

\section{INTRODUCTION}

Let $(M, g, J)$ be a connected, complex Hermitian manifold of complex dimension $n \geq 2$. We denote its fundamental 2-form by $\omega$; it is defined by $\omega(X, Y)=g(X, J Y)$. If there exists a real 1 -form $\theta$ satisfying the integrability condition

$$
d \omega=\theta \wedge \omega \quad \text { with } d \theta=0
$$

then $g$ is said to be a locally conformally Kähler (l.c.K.) metric. A complex manifold $M$ endowed with a l.c.K. metric is called a l.c.K. manifold. The conformal class of a l.c.K. metric $g$ is said to be a l.c.K. structure on $M$. The closed 1-form $\theta$ is called the Lee form and it encodes the geometric properties of such a manifold. The vector field $\theta^{\sharp}$, defined by $\theta(X)=g\left(X, \theta^{\sharp}\right)$, is called the Lee field.

The purpose of this paper is to study two kinds of transformation groups of a l.c.K. manifold $(M, g, J)$. We first consider Aut $_{\text {l.c.K. }}(M)$, the group of all conformal, holomorphic diffeomorphisms. We discuss its properties in $\S 2$. A holomorphic vector field $Z$ on $(M, g, J)$ generates a 1-dimensional complex Lie group $\mathcal{C}$. (The universal covering group of $\mathcal{C}$ is $\mathbb{C}$.) We call $\mathcal{C}$ a holomorphic flow on $M$.

Date: October 24, 2018.

1991 Mathematics Subject Classification. 53C55, 57S25.

Key words and phrases. Locally conformally Kähler manifold, Lee form, contact structure, strongly pseudoconvex CR-structure, $G$-structure, holomorphic complex torus action, transformation groups.

The second author is a member of EDGE, Research Training Network HPRN-CT-2000-00101, supported by The European Human Potential Programme. 
Definition 1.1. If a holomorphic flow $\mathcal{C}$ (resp. holomorphic vector field $Z$ ) belongs to $\operatorname{Aut}_{\text {l.c.K. }}(M)$ (resp. Lie algebra of $\operatorname{Aut}_{\text {l.c.K. }}(M)$ ), then $\mathcal{C}$ (resp. $Z$ ) is said to be a holomorphic l.c.K. flow (resp. holomorphic l.c.K. vector field).

A nontrivial subclass of l.c.K. manifolds is formed by those $(M, g, J)$ having parallel Lee form w.r.t. the Levi-Civita connection $\nabla^{g}$ (i.e. $\nabla^{g} \theta=0$ ). We observe that a compact nonKähler l.c.K. manifold $(M, g, J)$ with parallel Lee form $\theta$ supports a holomorphic vector field $Z=\theta^{\sharp}-i J \theta^{\sharp}$ which generates holomorphic isometries of $g$. (Compare [17], 118], [5].) We shall prove that the converse is also true:

Theorem A. Let $(M, g, J)$ be a compact, connected, l.c.K. non-Kähler manifold, of complex dimension at least 2. If $\operatorname{Aut}_{\text {l.c.K. }}(M)$ contains a holomorphic l.c.K. flow, then there exists a metric with parallel Lee form in the conformal class of $g$.

Corollary $\mathbf{A}_{1}$. With the same hypothesis, $M$ admits a l.c.K. metric with parallel Lee form if and only if it admits a holomorphic l.c.K. flow.

In $\S 3$, we discuss the existence of l.c.K. metrics with parallel Lee form on the Hopf manifold. (Compare with [6]). Let $\Lambda=\left(\lambda_{1}, \ldots, \lambda_{n}\right)$ with the $\lambda_{i}$ 's complex numbers satisfying $0<\left|\lambda_{n}\right| \leq \cdots \leq\left|\lambda_{1}\right|<1$. By a primary Hopf manifold $M_{\Lambda}$ of type $\Lambda$ we mean the compact quotient manifold of $\mathbb{C}^{n}-\{0\}$ by a subgroup $\Gamma_{\Lambda}$ generated by the transformation $\left(z_{1}, \ldots, z_{n}\right) \mapsto\left(\lambda_{1} z_{1}, \ldots, \lambda_{n} z_{n}\right)$. Note that a primary Hopf manifold of type $\Lambda$ of complex dimension 2 is a primary Hopf surface of Kähler rank 1 . We prove the following:

Theorem B. The primary Hopf manifold $M_{\Lambda}$ of type $\Lambda$ supports a l.c.K. metric with parallel Lee form.

More generally, we prove the existence of a l.c.K. metric with parallel Lee form on the Hopf manifold (cf. Theorem 3.1).

In the second half of the paper we adopt the viewpoint of $G$-structure theory in order to study a non-compact, non-holomorphic, transformation group of a compact l.c.K. manifold $(M, g, J)$. Locally, the 2 -form $\omega$ defines the real 1 -forms $\theta, \theta \circ J$ and $(n-1)$ complex 1 -forms $\theta^{\alpha}$ and their conjugates $\bar{\theta}^{\alpha}$, where $\theta \circ J$ is called the anti-Lee form and is defined by $\theta \circ$ $J(X)=\theta(J X)$. We consider the group $\operatorname{Aut}_{L C R}(M)$ of transformations of $M$ preserving the structure of unitary coframe fields $\mathcal{F}=\left\{\theta, \theta \circ J, \theta^{1}, \ldots, \theta^{n-1}, \bar{\theta}^{1}, \ldots, \bar{\theta}^{n-1}\right\}$. More precisely, an element $f$ of $\operatorname{Aut}_{L C R}(M)$ is called a Lee-Cauchy-Riemann (LCR) transformation if it satisfies the equations:

$$
\begin{aligned}
f^{*} \theta & =\theta, \\
f^{*}(\theta \circ J) & =\lambda \cdot(\theta \circ J), \\
f^{*} \theta^{\alpha} & =\sqrt{\lambda} \cdot \theta^{\beta} U_{\beta}^{\alpha}+(\theta \circ J) \cdot v^{\alpha}, \\
f^{*} \bar{\theta}^{\alpha} & =\sqrt{\lambda} \cdot \bar{\theta}^{\beta} \bar{U}_{\beta}^{\alpha}+(\theta \circ J) \cdot \bar{v}^{\alpha} .
\end{aligned}
$$

Here $\lambda$ is a positive, smooth function, and $v^{\alpha} \in \mathbb{C}, U_{\beta}^{\alpha} \in \mathrm{U}(n-1)$ are smooth functions. Obviously, if $\mathrm{I}(M, g, J)$ is the group of holomorphic isometries, then both $\mathrm{Aut}_{\text {l.c.K. }}(M)$ and $\operatorname{Aut}_{L C R}(M)$ contain $\mathrm{I}(M, g, J)$. 
As the main result of this part we exhibit the rigidity of compact l.c.K. manifolds under the existence of a non-compact LCR flow:

Theorem C. Let $(M, g, J)$ be a compact, connected, l.c.K. non-Kähler manifold of complex dimension at least 2 , with parallel Lee form $\theta$. Suppose that $M$ admits a closed subgroup $\mathbb{C}^{*}=S^{1} \times \mathbb{R}^{+}$of Lee-Cauchy-Riemann transformations whose $S^{1}$ subgroup induces the Lee field $\theta^{\sharp}$. Then $M$ is holomorphically isometric, up to scalar multiple of the metric, to the primary Hopf manifold $M_{\Lambda}$ of type $\Lambda$.

\section{LOCALLY CONFORMALly KÄHLER TRANSFORMATIONS}

Proposition 2.1. Let $(M, g, J)$ be a compact l.c.K. manifold with $\operatorname{dim}_{\mathbb{C}} M \geq 2$. Then $\operatorname{Aut}_{\text {l.c.K. }}(M)$ is a compact Lie group.

Proof. Note that $\operatorname{Aut}_{\text {l.c.K. }}(M)$ is a closed Lie subgroup in the group of all conformal diffeomorphisms of $(M, g)$. If $\operatorname{Aut}_{\text {l.c.K. }}(M)$ were noncompact, then by the celebrated result of Obata and Lelong-Ferrand ([14], [13]), $(M, g)$ would be conformally equivalent with the sphere $S^{2 n}, n \geq 2$. Hence $M$ would be simply connected. It is well known that a compact simply connected l.c.K. manifold is conformal to a Kähler manifold (cf. [5]), which is impossible because the sphere $S^{2 n}$ has no Kähler structure.

From now on, we shall suppose that the l.c.K. manifolds we work with are compact, nonKähler and, moreover, the Lee form is not identically zero at any point of the manifold. In particular, these manifolds are not simply connected (cf. [5]). Given a l.c.K. manifold $(M, g, J)$, let $\tilde{M}$ be the universal covering space of $M$, let $p: \tilde{M} \rightarrow M$ be the canonical projection and denote also by $J$ the lifted complex structure on $\tilde{M}$. We can associate to the fundamental 2-form $\omega$ a canonical Kähler form on $\tilde{M}$ as follows. Since the lee form $\theta$ is closed, its lift to $\tilde{M}$ is exact, hence $p^{*} \theta=d \tau$ for some smooth function $\tau$ on $\tilde{M}$. We put $h=e^{-\tau} \cdot p^{*} g$ (resp. $\left.\Omega=e^{-\tau} \cdot p^{*} \omega\right)$. It is easy to check that $d \Omega=0$, thus $h$ is a Kähler metric on $(\tilde{M}, J)$. In particular $g$ is locally conformal to the Kähler metric $h$ (compare with [5] and the bibliography therein). Let $f \in \operatorname{Aut}_{l . c . K .}(M)$. By definition, $f^{*} \omega=e^{\lambda} \cdot \omega$ for some function $\lambda$ on $M$. Differentiate this equality to yield that $\left(f^{*} \theta-\theta+d \lambda\right) \wedge \omega=0$. As $\omega$ is nondegenerate and $\operatorname{dim}_{\mathbb{C}} M>1, f^{*} \theta=\theta+d \lambda$. Since $p^{*} \theta=d \tau$, for any lift $\tilde{f}$ of $f$ to $\tilde{M}$ we have $d \tilde{f}^{*} \tau=d\left(\tau+p^{*} \lambda\right)$, thus $-\tilde{f}^{*} \tau+p^{*} \lambda=-\tau+c$ for some constant $c$. We can

write $\tilde{f}^{*} \Omega=e^{c} \cdot \Omega$. If $c \neq 0, \tilde{f}$ is a holomorphic homothety w.r.t. $h$; when $c=0, \tilde{f}$ will be an isometry.

We denote by $\mathcal{H}(\tilde{M}, \Omega, J)$ the group of all holomorphic, homothetic transformations of the universal cover $\tilde{M}$ w.r.t. the Kähler structure $(h, J)$. If $f_{1}, f_{2} \in \mathcal{H}(\tilde{M}, \Omega, J)$, there exists some constant $\rho\left(f_{i}\right)(i=1,2)$ satisfying $f_{i}^{*} \Omega=\rho\left(f_{i}\right) \cdot \Omega$ as above. It is easy to check that $\rho\left(f_{1} \circ f_{2}\right)=\rho\left(f_{1}\right) \cdot \rho\left(f_{2}\right)$. We obtain a continuous homomorphism:

$$
\rho: \mathcal{H}(\tilde{M}, \Omega, J) \longrightarrow \mathbb{R}^{+}
$$


Let $\pi_{1}(M)$ be the fundamental group of $M$. Then we note that $\pi_{1}(M) \subset \mathcal{H}(\tilde{M}, \Omega, J)$. For this, if $\gamma \in \pi_{1}(M)$, then $\gamma^{*} \Omega=e^{-\gamma^{*} \tau} \cdot \gamma^{*} p^{*} \omega=e^{-\gamma^{*} \tau} \cdot p^{*} \omega=e^{-\gamma^{*} \tau+\tau} \cdot \Omega$. Since $\Omega$ is a Kähler form $(n \geq 2), e^{-\gamma^{*} \tau+\tau}$ must be constant $\rho(\gamma)$.

Let $\mathcal{C}$ be a holomorphic l.c.K. flow on $M$. If we denote $\tilde{\mathcal{C}}$ a lift of $\mathcal{C}$ to $\tilde{M}$, then $\tilde{\mathcal{C}} \subset$ $\mathcal{H}(\tilde{M}, \Omega, J)$. If $V$ is a vector field which generates a one-parameter subgroup of $\tilde{\mathcal{C}}$, then so does $J V$ such as $V$ and $J V$ together generate $\tilde{\mathcal{C}}$. We define a smooth function $s$ : $\tilde{M} \rightarrow \mathbb{R}$ to be $s(x)=\Omega\left(J V_{x}, V_{x}\right)$. Since $\tilde{\mathcal{C}}$ centralizes each element $\gamma$ of $\pi_{1}(M)$, it follows that $s(\gamma x)=\Omega\left(J V_{\gamma x}, V_{\gamma x}\right)=\Omega\left(\gamma_{*} J V_{x}, \gamma_{*} V_{x}\right)=\rho(\gamma) s(x)$. If every element $\gamma$ satisfies that $\rho(\gamma)=1$, i.e., $\gamma^{*} \Omega=\Omega$, then $\pi_{1}(M)$ acts as holomorphic isometries of $h$ so that $\Omega$ would induce a Kähler structure on $M$. By our hypothesis, this does not occur. There exists at least one element $\gamma$ such that $\rho(\gamma) \neq 1$. In particular, we note that:

The function $s$ is not constant on $\tilde{M}$.

On the other hand, we prove the following lemma. (The proof of the lemma is almost same as that of [9].)

Lemma 2.1. $\rho(\tilde{\mathcal{C}})=\mathbb{R}^{+}$, i.e., the group $\tilde{\mathcal{C}}$ acts by holomorphic, non-trivial homotheties w.r.t. the Kähler metric $h$ on $\tilde{M}$.

Proof. Suppose that $\rho(\tilde{\mathcal{C}})=\{1\}$. Then $\tilde{\mathcal{C}}$ leaves $\Omega$ invariant. As $\{V, J V\}$ generates $\tilde{\mathcal{C}}$, it follows that $\mathcal{L}_{V} \Omega=\mathcal{L}_{J V} \Omega=0$. In particular, $V s=(J V) s=0$. For any distribution $D$ on $\tilde{M}$, denote by $D^{\perp}$ the orthogonal complement to $D$ w.r.t. the metric $h$ where $h(\tilde{X}, \tilde{Y})=$ $\Omega(J \tilde{X}, \tilde{Y})$. Since $0=\left(\mathcal{L}_{V} \Omega\right)(J V, \tilde{X})=V \Omega(J V, \tilde{X})-\Omega([V, J V], \tilde{X})-\Omega(J V,[V, \tilde{X}])$, if $\tilde{X} \in\{V, J V\}^{\perp}$, then $\Omega(J V,[V, \tilde{X}])=0$, similarly $\Omega(V,[J V, \tilde{X}])=0$. The equality

$$
\begin{aligned}
0=3 d \Omega(\tilde{X}, V, J V)= & \tilde{X} \Omega(V, J V)-V \Omega(\tilde{X}, J V)+J V \Omega(\tilde{X}, V) \\
& -\Omega([\tilde{X}, V], J V)-\Omega([V, J V], \tilde{X})-\Omega([J V, \tilde{X}], V)
\end{aligned}
$$

implies that $\tilde{X} \Omega(V, J V)=0$, i.e., $\tilde{X} s=0$ for any $\tilde{X} \in\{V, J V\}^{\perp}$. Therefore, $s$ becomes constant, being a contradiction to (2.2).

2.1. The submanifold $W$ and its pseudo-Hermitian structure. As Ker $\rho$ has one dimension, denote by $-J \xi$ the vector field whose one-parameter subgroup $\left\{\psi_{t}\right\}_{t \in \mathbb{R}}$ acts as holomorphic isometries on $\tilde{M}$.

$$
\psi_{t}^{*} \Omega=\Omega, \quad t \in \mathbb{R} .
$$

Since $-J \xi$ and $\xi$ together generate the group $\tilde{\mathcal{C}}$, the 1-parameter subgroup $\left\{\varphi_{t}\right\}_{t \in \mathbb{R}}$ generated by $\xi$ acts as nontrivial holomorphic homotheties w.r.t. $\Omega$ by Lemma 2.1. In particular, the group $\left\{\varphi_{t}\right\}_{t \in \mathbb{R}}$ is isomorphic to $\mathbb{R}$. Since $\varphi_{t}^{*} \Omega=\rho\left(\varphi_{t}\right) \cdot \Omega\left(t \in \mathbb{R}, \rho\left(\varphi_{t}\right) \in \mathbb{R}^{+}\right)$from (2.1) and $\rho$ is a continuous homomorphism, $\rho\left(\varphi_{t}\right)=e^{a t}$ for some constant $a \neq 0$. We may normalize $a=1$ so that:

$$
\varphi_{t}^{*} \Omega=e^{t} \cdot \Omega, \quad t \in \mathbb{R}
$$


Lemma 2.2. The group $\left\{\varphi_{t}\right\}_{t \in \mathbb{R}}$ acts properly and hence freely on $\tilde{M}$. In particular, $\xi \neq 0$ everywhere on $\tilde{M}$.

Proof. Recall that $\mathcal{C}$ lies in $\operatorname{Aut}_{\text {l.c.K. }}(M)$ by definition. As $\operatorname{Aut}_{\text {l.c.K. }}(M)$ is a compact Lie group, its closure $\overline{\mathcal{C}}$ in $\operatorname{Aut}_{\text {l.c.K. }}(M)$ is also compact and so isomorphic to a $k$-torus $(k \geq 2)$. Therefore, the lift $H$ of $\overline{\mathcal{C}}$ to $\tilde{M}$ acts properly on $\tilde{M}$. The lift $H$ is isomorphic to $\mathbb{R}^{\ell} \times T^{m}$ where $\ell+m=k$. Note that $\ell \geq 1$ because $\rho$ maps any compact subgroup of $H$ to $\{1\}$, but the group $\left\{\varphi_{t}\right\}_{t \in \mathbb{R}} \subset H$ satisfies $\rho\left(\left\{\varphi_{t}\right\}\right)=\mathbb{R}^{+}$. Hence the group $\left\{\varphi_{t}\right\}_{t \in \mathbb{R}}$ has a nontrivial summand in $\mathbb{R}^{\ell}$ which implies that $\left\{\varphi_{t}\right\}_{t \in \mathbb{R}}$ is closed in $H$. Thus, the group $\left\{\varphi_{t}\right\}_{t \in \mathbb{R}}$ acts properly on $\tilde{M}$. If we note that $\left\{\varphi_{t}\right\}_{t \in \mathbb{R}}$ is isomorphic to $\mathbb{R}$, then it acts freely on $\tilde{M}$.

Proposition 2.2. Let $s: \tilde{M} \rightarrow \mathbb{R}$ be the smooth map defined as $s(x)=\Omega\left(J \xi_{x}, \xi_{x}\right)$. Then 1 is a regular value of $s$, hence $s^{-1}(1)$ is a codimension one, regular submanifold of $\tilde{M}$.

Proof. As $\varphi_{t}$ is holomorphic, $s\left(\varphi_{t} x\right)=\Omega\left(J \xi_{\varphi_{t} x}, \xi_{\varphi_{t} x}\right)=\Omega\left(\varphi_{t *} J \xi_{x}, \varphi_{t *} \xi_{x}\right)=e^{t} \cdot s(x)$. Hence,

$$
\mathcal{L}_{\xi} s=\lim _{t \rightarrow 0} \frac{\varphi_{t}^{*} s-s}{t}=s .
$$

We note also that

$$
\mathcal{L}_{\xi} \Omega=\Omega
$$

By Lemma 2.2, notice that $\xi \neq 0$ everywhere on $\tilde{M}$. Since $s(x) \neq 0, s^{-1}(1) \neq \emptyset$. For $x \in s^{-1}(1), d s\left(\xi_{x}\right)=\left(\mathcal{L}_{\xi} s\right)(x)=s(x)=1$. This proves that $d s: T_{x} \tilde{M} \rightarrow \mathbb{R}$ is onto and so $s^{-1}(1)$ is a codimension one smooth regular submanifold of $\tilde{M}$.

Let now $W=s^{-1}(1)$. We can prove:

Lemma 2.3. The submanifold $W$ is connected and the map $H: \mathbb{R} \times W \rightarrow \tilde{M}$, defined by $H(t, w)=\varphi_{t} w$ is an equivariant diffeomorphism.

Proof. Let $W_{0}$ be a component of $s^{-1}(1)$ and $\mathbb{R} \cdot W_{0}$ be the set $\left\{\varphi_{t} w ; w \in W_{0}, t \in \mathbb{R}\right\}$. As $\mathbb{R}=\left\{\varphi_{t}\right\}$ acts freely and $s\left(\varphi_{t} x\right)=e^{t} s(x)$, we have $\varphi_{t} W_{0} \cap W_{0}=\emptyset$ for $t \neq 0$. Thus $\mathbb{R} \cdot W_{0}$ is an open subset of $\tilde{M}$. We prove that it is also closed. Let $\overline{\mathbb{R} \cdot W_{0}}$ be the closure of $\mathbb{R} \cdot W_{0}$ in $\tilde{M}$. We choose a limit point $p=\lim \varphi_{t_{i}} w_{i} \in \overline{\mathbb{R} \cdot W_{0}}$. Then $s(p)=\lim s\left(\varphi_{t_{i}} w_{i}\right)=\lim e^{t_{i}} s\left(w_{i}\right)=$ $\lim e^{t_{i}}$. Put $t=\log s(p)$, then $t=\lim t_{i}$, so $\varphi_{t}^{-1}(p)=\lim \varphi_{t_{i}}^{-1}\left(\lim \varphi_{t_{i}} w_{i}\right)=\lim w_{i}$. Since $s^{-1}(1)$ is regular (i.e. closed w.r.t. the relative topology induced from $\tilde{M}$ ), its component $W_{0}$ is also closed. Hence $\varphi_{t}^{-1} p \in W_{0}$. Therefore $p=\varphi_{t}\left(\varphi_{t}^{-1} p\right) \in \mathbb{R} \cdot W_{0}$, proving that $\mathbb{R} \cdot W_{0}$ is closed in $\tilde{M}$. In conclusion, $\mathbb{R} \cdot W_{0}=\tilde{M}$. Now, if $W_{1}$ is another component of $s^{-1}(1)$, the same argument shows $\mathbb{R} \cdot W_{1}=\tilde{M}$. As $\mathbb{R} \cdot W_{0}=\mathbb{R} \cdot W_{1}$ and $s\left(W_{1}\right)=1$, this implies $W_{0}=W_{1}$, in other words $W$ is connected. 
Let $i: W \rightarrow \tilde{M}$ be the inclusion and $\pi: \tilde{M} \rightarrow W$ be the canonical projection. Define a 1-form $\eta$ on $W$ to be

$$
\eta=i^{*} \iota_{\xi} \Omega
$$

Here $\iota_{\xi}$ denotes the interior product with $\xi$. We have from $\S 2.1$ that:

$$
\left.\frac{d \psi_{t}}{d t}(x)\right|_{t=0}=-J \xi_{x}
$$

Using (2.3), $s\left(\psi_{t} w\right)=s(w)=1(w \in W)$ so that the group $\left\{\psi_{t}\right\}_{t \in \mathbb{R}}$ leaves $W$ invariant. Hence, the vector field $-J \xi$ restricts to a vector field $A$ to $W$. If $\left\{\psi_{t}^{\prime}\right\}_{t \in \mathbb{R}}$ is the oneparameter subgroup generated by $A$, then

$$
\psi_{t}=i \circ \psi_{t}^{\prime}
$$

Lemma 2.4. The 1-form $\eta$ is a contact form on $W$ for which $A$ is the characteristic vector field (Reeb field).

Proof. First note that $\eta\left(A_{w}\right)=\iota_{\xi} \Omega\left(-J \xi_{w}\right)=\Omega\left(J \xi_{w}, \xi_{w}\right)=s(w)=1 \quad(w \in W)$. Moreover, from (2.5), $d \eta=i^{*} d_{\xi} \Omega=i^{*}\left(d \iota_{\xi} \Omega+\iota_{\xi} d \Omega\right)=i^{*} \mathcal{L}_{\xi} \Omega=i^{*} \Omega$. Hence, $\eta \wedge d \eta^{n-1} \neq 0$ on $W$ showing that $\eta$ is a contact form. Noting (2.3), (2.8) and that both $\varphi_{t}$ and $\psi_{\theta}$ commutes each other, it is easy to see that

$$
\begin{gathered}
\psi_{t^{\prime} \iota_{\xi} \Omega}=\iota_{\xi} \Omega \quad \text { on } \tilde{M} . \\
\psi_{t}^{\prime *} \eta=\eta \quad \text { on } W .
\end{gathered}
$$

Let Null $\eta=\{X \in T W \mid \eta(X)=0\}$ be the contact subbundle. Since $\mathcal{L}_{A} \eta(X)=A \eta(X)-$ $\eta([A, X])$ and $\mathcal{L}_{A} \eta=0$ from (2.9), if $X \in$ Null $\eta$, then $\eta([A, X])=0$. Moreover,

$d \eta(A, X)=\frac{1}{2}(A \eta(X)-X \eta(A)-\eta([A, X]))=0$, which implies that $d \eta(A, X)=0$ for all $X \in T W$, showing that $A$ is the characteristic vector field.

Recall that $\mathbb{R} \rightarrow \tilde{M} \stackrel{\pi}{\rightarrow} W$ is a principal fiber bundle with $T \mathbb{R}=<\xi>$. By Lemma 2.3, each point $x \in \tilde{M}$ can be described uniquely as $x=\varphi_{t} w$. Using (2.8),

$$
\begin{gathered}
\pi \circ \psi_{\theta}(x)=\pi \circ \psi_{\theta}\left(\varphi_{t} w\right)=\pi \circ \varphi_{t}\left(\psi_{\theta} w\right) \\
=\pi \circ i \psi_{\theta}^{\prime}(w)=\psi_{\theta}^{\prime}(w)=\psi_{\theta}^{\prime} \circ \pi(x),
\end{gathered}
$$

hence, $\pi_{*}(-J \xi)=A$. As $i_{*} \pi_{*} X_{x}-X_{x}=a \cdot \xi_{x}$ for some function $a$, using (2.6), $\pi$ maps $\{\xi, J \xi\}^{\perp}$ isomorphically onto Null $\eta$. Since $\{\xi, J \xi\}^{\perp}$ is $J$-invariant, there exists an almost complex structure $J$ on Null $\eta$ such that the following diagram is commutative:

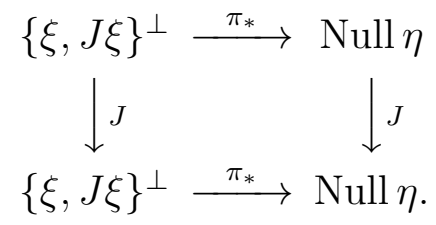


Proposition 2.3. The pair $(\eta, J)$ is a strictly pseudoconvex, pseudo-Hermitian structure on $\tilde{W}$.

Proof. Let $\Psi:$ Null $\eta \times$ Null $\eta \rightarrow \mathbb{R}$ be the bilinear form defined by $\Psi(X, Y)=d \eta(J X, Y)$. There exist $\tilde{X}, \tilde{Y} \in\{\xi, J \xi\}^{\perp}$ such that $\pi_{*} \tilde{X}=X, \pi_{*} \tilde{Y}=Y$. Then it is easy to see that $i_{*} J X \equiv J \tilde{X}, \quad i_{*} Y \equiv \tilde{Y} \bmod \xi$. Using $d \eta=i^{*} \Omega$ as above, $\Psi(X, Y)=i^{*} \Omega(J X, Y)=$ $\Omega(J \tilde{X}, \tilde{Y})=h(\tilde{X}, \tilde{Y})$, hence $\Psi$ is positive definite. By definition, $\eta$ is strictly pseudoconvex. Let $\{\xi, J \xi\}^{\perp} \otimes \mathbb{C}=B^{1,0} \oplus B^{0,1}$ be the canonical splitting of $J$. Then we prove that $\left[B^{1,0}, B^{1,0}\right] \subset B^{1,0}$. Let $\tilde{X}, \tilde{Y} \in B^{1,0}$. Since $T^{1,0} \tilde{M}=\{\xi-\mathrm{i} J \xi\} \oplus B^{1,0}$ and $J$ is integrable on $\tilde{M}$, $[\tilde{X}, \tilde{Y}] \in T^{1,0} \tilde{M}$. Put $[\tilde{X}, \tilde{Y}]=a(\xi-\mathrm{i} J \xi)+\tilde{Z}$ for some function $a$ and $\tilde{Z} \in B^{1,0}$. As $\pi_{*}(-J \xi)=A$ from $(2.10), \pi_{*}([\tilde{X}, \tilde{Y}])=a \mathrm{i} A+\pi_{*} \tilde{Z}$. By definition, $2 d \eta\left(\pi_{*} \tilde{X}, \pi_{*} \tilde{Y}\right)=-\eta\left(\left[\pi_{*} \tilde{X}, \pi_{*} \tilde{Y}\right]\right)=-a \mathrm{i}$. On the other hand, since $\Omega$ is $J$-invariant, $\Omega(\tilde{X}, \tilde{Y})=0$ for $\forall \tilde{X}, \tilde{Y} \in B^{1,0}$. As above, $i_{*} \pi_{*} \tilde{X} \equiv \tilde{X} \bmod \xi$, similarly for $\tilde{Y}$, we obtain that $d \eta\left(\pi_{*} \tilde{X}, \pi_{*} \tilde{Y}\right)=\Omega\left(i_{*} \pi_{*} \tilde{X}, i_{*} \pi_{*} \tilde{Y}\right)=\Omega(\tilde{X}, \tilde{Y})=0$. Hence, $a=0$ and so $[\tilde{X}, \tilde{Y}]=\tilde{Z} \in B^{1,0}$. If we note that $\pi_{*}:\{\xi, J \xi\}^{\perp} \otimes \mathbb{C} \rightarrow$ Null $\eta \otimes \mathbb{C}$ is $J$-isomorphic by (2.11), then Null $\eta \otimes \mathbb{C}=\pi_{*} B^{1,0} \oplus \pi_{*} B^{0,1}$ is the splitting for $J$, in which we have shown $\left[\pi_{*} B^{1,0}, \pi_{*} B^{1,0}\right] \subset \pi_{*} B^{1,0}$. Therefore $J$ is a complex structure on Null $\eta$.

Consider the group of pseudo-Hermitian transformations on $(W, \eta, J)$ :

$$
\operatorname{PSH}(W, \eta, J)=\left\{f \in \operatorname{Diff}(W) \mid f^{*} \eta=\eta, f_{*} \circ J=J \circ f_{*} \text { on Null } \eta\right\} .
$$

Corollary 2.1. The characteristic vector field A generates the subgroup $\left\{\psi_{t}^{\prime}\right\}_{t \in \mathbb{R}}$ consisting of pseudo-Hermitian transformations.

Proof. By (2.3) and (2.9), $\psi_{t}$ (resp. $\left.\psi_{t}^{\prime}\right)$ preserves $\{\xi, J \xi\}^{\perp}$ (resp. Null $\eta$ ). Then the equality $\pi \circ \psi_{\theta}=\psi_{\theta}^{\prime} \circ \pi$ from (2.10) with diagram (2.11) implies that $\psi_{t *}^{\prime} J=J \psi_{t *}^{\prime}$ on Null $\eta$. Therefore

$$
\left\{\psi_{t}^{\prime}\right\}_{t \in \mathbb{R}} \subset \operatorname{PSH}(W, \eta, J) .
$$

\section{Proof of Theorem A.}

2.2. Parallel Lee form. Let $Y_{\varphi_{t} w} \in T_{\varphi_{t} w} \tilde{M}$ be any vector field. As $\pi_{*} Y_{\varphi_{t} w} \in T_{w} W$, $i_{*} \pi_{*} Y_{\varphi_{t} w}-\varphi_{-t_{*}} Y_{\varphi_{t} w}=\lambda \xi_{w}$ for some function $\lambda$. Then,

$$
\begin{aligned}
& \iota_{\xi} \Omega\left(i_{*} \pi_{*} Y_{\varphi_{t} w}\right)=\Omega\left(\xi_{w}, i_{*} \pi_{*} Y_{\varphi_{t} w}\right)=\Omega\left(\xi_{w}, \varphi_{-t_{*}} Y_{\varphi_{t} w}\right)+\Omega\left(\xi_{w}, \lambda \xi_{w}\right) \\
& =\varphi_{-t}^{*} \Omega\left(\varphi_{t_{*}} \xi_{w}, Y_{\varphi_{t} w}\right)=e^{-t} \Omega\left(\xi_{\varphi_{t} w}, Y_{\varphi_{t} w}\right)=e^{-t} \iota_{\xi} \Omega\left(Y_{\varphi_{t} w}\right) .
\end{aligned}
$$

By definition (2.6),

$$
\pi^{*} \eta=\pi^{*} i^{*} \iota_{\xi} \Omega=e^{-t} \iota_{\xi} \Omega, \text { equivalently, } e^{t} \pi^{*} \eta=\iota_{\xi} \Omega .
$$

As $\Omega=\mathcal{L}_{\xi} \Omega=d \iota_{\xi} \Omega$ from (2.5), we obtain that

$$
d\left(e^{t} \pi^{*} \eta\right)=\Omega \text { on } \tilde{M}
$$


For the given l.c.K. metric $g$, the Kähler metric $h$ is obtained as $h=e^{-\tau} \cdot p^{*} g$ where $d \tau=\tilde{\theta}$. As $\omega$ is the fundamental 2 -form of $g$, note that $\Omega=e^{-\tau} \cdot p^{*} \omega$.

We now consider on $\tilde{M}$ the 2-form:

$$
\bar{\Theta}=2 e^{-t} \cdot d\left(e^{t} \pi^{*} \eta\right)\left(=2 e^{-t} \cdot \Omega\right)
$$

Then $\bar{g}(X, Y)=\bar{\Theta}(J X, Y)$ is a l.c.K. metric. Put $\bar{\theta}=-d t$. Then, as $d \bar{\Theta}=-2 e^{-t} d t \wedge d\left(e^{t} \pi^{*} \eta\right)=-d t \wedge \bar{\Theta}$, so $\bar{\theta}$ is the Lee form of $\bar{g}$.

Lemma 2.5. $\bar{\theta}$ is parallel w.r.t. $\bar{g}\left(\nabla^{\bar{g}} \bar{\theta}=0\right)$.

Proof. First we determine the Lee field $\bar{\theta}^{\sharp} .\left(\bar{\theta}(X)=\bar{g}\left(X, \bar{\theta}^{\sharp}\right)\right.$.) We start from:

$$
\begin{aligned}
\bar{g}(\xi, Y) & =\bar{\Theta}(J \xi, Y)=2 e^{-t}\left(e^{t} d t \wedge \pi^{*} \eta+e^{t} d \pi^{*} \eta\right)(J \xi, Y) \\
& =2\left(d t \wedge \pi^{*} \eta+d \pi^{*} \eta\right)(J \xi, Y)=2\left(d t \wedge \pi^{*} \eta\right)(J \xi, Y)
\end{aligned}
$$

because $A=-\pi_{*} J \xi$ is the characteristic vector field of the contact form $\eta$. As before, a point $x \in \tilde{M}$ can be described uniquely as $\varphi_{t} w$ for some $w \in W$. In particular, using Lemma 2.3, the $t$-coordinate of $x$ is $t$. Noting that $\psi_{\theta}(x)=\varphi_{t} \psi_{\theta} w$ and $\psi_{\theta} w \in W$, by uniqueness the $t$-coordinate of $\psi_{\theta}(x), t\left(\psi_{\theta}(x)\right)=t$. From (2.7),

$$
d t\left(-J \xi_{x}\right)=d t\left(\left.\frac{d \psi_{\theta}}{d \theta}(x)\right|_{\theta=0}\right)=\left.\frac{d t}{d \theta}\right|_{\theta=0}=0 .
$$

The above formula becomes:

$$
\bar{g}(\xi, Y)=2\left(d t \wedge \pi^{*} \eta\right)(J \xi, Y)=-d t(Y) \eta(-A)=d t(Y)=-\bar{\theta}(Y)=-\bar{g}\left(Y, \bar{\theta}^{\sharp}\right)
$$

proving that $\bar{\theta}^{\sharp}=-\xi$. Next we observe that the flow $\left\{\varphi_{s}\right\}_{s \in \mathbb{R}}$ acts by isometries w.r.t. $\bar{g}$. As $\varphi_{s}$ is holomorphic, it is enough to prove that each $\varphi_{s}$ leaves $\bar{\Theta}$ invariant. But

$$
\varphi_{s}^{*} \bar{\Theta}=2 e^{-\varphi_{s}^{*} t} d\left(e^{\varphi_{s}^{*} t} \varphi_{s}^{*} \pi^{*} \eta\right)=2 e^{-(s+t)} d\left(e^{s+t} \pi^{*} \eta\right)=2 e^{-t} d\left(e^{t} \pi^{*} \eta\right)=\bar{\Theta} .
$$

Thus $\mathcal{L}_{\theta^{\sharp}} \bar{g}=-\mathcal{L}_{\xi} \bar{g}=0$. Now we put $\sigma=\bar{\theta}$ in the equality $\left(\mathcal{L}_{\sigma^{\sharp}} \bar{g}\right)(X, Y)+2 d \sigma(X, Y)=$ $2 \bar{g}\left(\nabla_{X}^{\bar{g}} \sigma^{\sharp}, Y\right)$, valid for any 1 -form $\sigma$, take into account $d \bar{\theta}=0$ and obtain $\nabla^{\bar{g}} \bar{\theta}^{\sharp}=0$ which is equivalent with $\nabla^{\bar{g}} \bar{\theta}=0$, so $\bar{\theta}$ is parallel w.r.t. $\bar{g}$ as announced.

By equation (2.16), $\bar{g}$ is conformal to the lifted metric $p^{*} g$ :

$$
\bar{\Theta}=\mu \cdot p^{*} \omega \quad \text { (equivalently } \bar{g}=\mu \cdot p^{*} g \text { ) }
$$

where $\mu=2 e^{-(t+\tau)}: \tilde{M} \rightarrow \mathbb{R}^{+}$is a smooth map. We finally prove:

Lemma 2.6. $\pi_{1}(M)$ acts by holomorphic isometries of $\bar{g}$. In particular, $\pi_{1}(M)$ leaves $\bar{\theta}$ invariant.

Proof. We prove the following two facts:

1. $\gamma^{*} \pi^{*} \eta=\pi^{*} \eta$ for every $\gamma \in \pi_{1}(M)$.

2. $\gamma^{*} e^{t}=\rho(\gamma) \cdot e^{t}$ where $\rho: \pi_{1}(M) \rightarrow \mathbb{R}^{+}$is the homomorphism as before. 
First note that as $\mathbb{R}=\left\{\varphi_{t}\right\}$ centralizes $\pi_{1}(M), \gamma_{*} \xi=\xi$ for $\gamma \in \pi_{1}(M)$. As $\gamma$ is holomorphic, $\gamma_{*} J \xi=J \xi$. Since $\pi_{1}(M)$ acts on $\tilde{M}$ as holomorphic homothetic transformations, (i.e., $\gamma^{*} \Omega=\rho(\gamma) \cdot \Omega$ ), $\pi_{1}(M)$ preserves $\{\xi, J \xi\}^{\perp}$. If we recall that $\pi_{*}:\{\xi, J \xi\}^{\perp} \rightarrow$ Null $\eta$ is isomorphic, then for $X \in\{\xi, J \xi\}^{\perp}, \gamma^{*} \pi^{*} \eta(X)=\eta\left(\pi_{*} \gamma_{*} X\right)=0$. As $-\pi_{*} J \xi=A$ is characteristic, it follows $\gamma^{*} \pi^{*} \eta(J \xi)=\eta\left(\pi_{*} \gamma_{*} J \xi\right)=\eta\left(\pi_{*} J \xi\right)=-1$. This shows that $\gamma^{*} \pi^{*} \eta=\pi^{*} \eta$ on $\tilde{M}$. On the other hand, if we note $\gamma_{*} \xi=\xi$, then

$$
\begin{aligned}
\gamma^{*}\left(\iota_{\xi} \Omega\right)(X) & =\Omega\left(\xi, \gamma_{*} X\right)=\Omega\left(\gamma_{*} \xi, \gamma_{*} X\right)=\gamma^{*} \Omega(\xi, X) \\
& =\rho(\gamma) \cdot \Omega(\xi, X)=\rho(\gamma) \cdot \iota_{\xi} \Omega(X)
\end{aligned}
$$

where $\rho(\gamma)$ is a positive constant number. Applying $\gamma^{*}$ to $\pi^{*} \eta=e^{-t} \cdot \iota_{\xi} \Omega$ from (2.14), we obtain $\gamma^{*} e^{-t} \cdot \rho(\gamma)=e^{-t}$. Equivalently, $\gamma^{*} e^{t}=\rho(\gamma) \cdot e^{t}$. This shows 1 and 2 .

From (2.16),

$$
\begin{aligned}
\gamma^{*} \bar{\Theta} & =\gamma^{*}\left(2 e^{-t} \cdot d\left(e^{t} \pi^{*} \eta\right)\right)=2 \rho(\gamma)^{-1} \cdot e^{-t} d\left(\rho(\gamma) \cdot e^{t} \gamma^{*} \pi^{*} \eta\right) \\
& =2 e^{-t} \cdot d\left(e^{t} \pi^{*} \eta\right)=\bar{\Theta} .
\end{aligned}
$$

Since $\bar{g}(X, Y)=\bar{\Theta}(J X, Y), \pi_{1}(M)$ acts through holomorphic isometries of $\bar{g}$. We have that $\bar{\theta}(Y)=\bar{g}\left(Y, \bar{\theta}^{\sharp}\right)=-\bar{g}(Y, \xi)(Y \in T \tilde{M})$ from (2.18). Then,

$$
\gamma^{*} \bar{\theta}(Y)=-\bar{g}\left(\gamma_{*} Y, \xi\right)=-\bar{g}\left(\gamma_{*} Y, \gamma_{*} \xi\right)=-\bar{g}(Y, \xi)=\bar{\theta}(Y) .
$$

From this lemma, the covering map $p: \tilde{M} \rightarrow M$ induces a l.c.K. metric $\hat{g}$ with parallel Lee form $\hat{\theta}$ on $M$ such that $p^{*} \hat{g}=\bar{g}$ and $p^{*} \hat{\theta}=\bar{\theta}$ with $\nabla_{p_{*} X}^{\hat{g}} \hat{\theta}\left(p_{*} Y\right)=\nabla_{X}^{\bar{g}} \bar{\theta}(Y)$. Applying $\gamma^{*}$ to the both side of (2.19), we derive

$$
\begin{aligned}
& \gamma^{*} \bar{g}=\bar{g}=\mu \cdot p^{*} g . \\
& \gamma^{*} \mu \cdot \gamma^{*} p^{*} g=\gamma^{*} \mu \cdot p^{*} g .
\end{aligned}
$$

Therefore $\gamma^{*} \mu=\mu$ which implies that $\mu$ factors through a map $\hat{\mu}: M \rightarrow \mathbb{R}^{+}$so that $p^{*} \hat{g}=p^{*}(\hat{\mu} \cdot g)$. We have $\hat{\mu} \cdot g=\hat{g}$. The conformal class of $g$ contains a l.c.K. metric $\hat{g}$ with parallel Lee form $\hat{\theta}$. This finishes the proof of Theorem A.

As to Corollary $\mathrm{A}_{1}$ in the Introduction, we recall the following. (Compare [17], [5, p.37].) Let $(M, g, J)$ be a compact, connected, non-Kähler, l.c.K. manifold with parallel Lee form $\theta$. Then the following results hold: $g\left(\theta^{\sharp}, \theta^{\sharp}\right)=$ const,

$$
\begin{aligned}
& \mathcal{L}_{\theta^{\sharp}} J=\mathcal{L}_{J \theta^{\sharp}} J=0, \\
& \mathcal{L}_{\theta^{\sharp}} g=\mathcal{L}_{J \theta^{\sharp}} g=0 .
\end{aligned}
$$

Then $Z=\theta^{\sharp}-i J \theta^{\sharp}$ is a holomorphic vector field because $\left[\theta^{\sharp}, J \theta^{\sharp}\right]=0$ (cf. $[1]$ ). By Definition 1.1, $Z=\theta^{\sharp}-i J \theta^{\sharp}$ is a holomorphic l.c.K. vector field.

Proposition 2.4. The real vector fields $\theta^{\sharp}$ and $J \theta^{\sharp}$ satisfy the following:

1. A flow generated by the Lee field $\theta^{\sharp}$ lifts to a one-parameter subgroup of nontrivial homothetic holomorphic transformations w.r.t. $\Omega$. 
2. A flow generated by the anti-Lee field $-J \theta^{\sharp}$ lifts to a one-parameter subgroup consisting of holomorphic isometries w.r.t. $\Omega$.

Proof. Let $\left\{\hat{\varphi}_{t}\right\}_{t \in \mathbb{R}}$ be the flow generated by $\theta^{\sharp}$ on $M$ and $\left\{\varphi_{t}\right\}_{t \in \mathbb{R}}$ its lift to $\tilde{M}$. Denote by $\xi$ the vector field on $\tilde{M}$ induced by $\left\{\varphi_{t}\right\}$. Then, $p_{*} \xi=\theta^{\sharp}$. Because $\theta$ is parallel, $\left\{\hat{\varphi}_{t}\right\}$ (resp. $\left.\left\{\varphi_{t}\right\}\right)$ acts by holomorphic isometries w.r.t. $g$ (resp. $p^{*} g$ ). In particular, $\left\{\varphi_{t}\right\}$ preserves $p^{*} \omega$. Then, for $\Omega=e^{-\tau} p^{*} \omega$, we have $\varphi_{t}^{*} \Omega=e^{-\left(\varphi_{t}^{*} \tau-\tau\right)} \Omega$. As $\rho:\left\{\varphi_{t}\right\} t \in \mathbb{R} \rightarrow \mathbb{R}^{+}$is a homomorphism and $\rho\left(\varphi_{t}\right)=e^{-\left(\varphi_{t}^{*} \tau-\tau\right)}$ is a constant for each $t \in \mathbb{R}\left(\operatorname{dim}_{\mathbb{C}} M \geq 2\right)$, we can describe as $-\left(\varphi_{t}^{*} \tau-\tau\right)=c \cdot t$ for some constant $c$. Recall that $h$ is the Kähler metric associated to $\Omega$. If $\left\{\varphi_{t}\right\}$ acts as holomorphic isometries w.r.t. $h$, then the above equation implies that $c=0$, i.e. $\varphi_{t}^{*} \tau-\tau=0$ for every $t$, and so $\mathcal{L}_{\xi} \tau=0$. On the other hand, as $d \tau=p^{*} \theta$, we have:

$$
0=\mathcal{L}_{\xi} \tau=d \tau(\xi)=\theta\left(p_{*} \xi\right)=\theta\left(\theta^{\sharp}\right)=\text { const }>0,
$$

being a contradiction. Thus, $\varphi_{t}^{*} \Omega=\rho\left(\varphi_{t}\right) \Omega=e^{c \cdot t} \Omega$ with $c \neq 0$. Hence, $\left\{\varphi_{t}\right\}_{t \in \mathbb{R}}$ is a group of nontrivial homothetic holomorphic transformations isomorphic to $\mathbb{R}$. On the other hand, let $\left\{\hat{\psi}_{t}\right\}_{t \in \mathbb{R}}$ (resp. $\left.\left\{\psi_{t}\right\}_{t \in \mathbb{R}}\right)$ be the flow generated by $-J \theta^{\sharp}$ on $M$ (resp. $-J \xi$ on $\tilde{M}$ ). As $p_{*}(J \xi)=J p_{*} \xi=J \theta^{\sharp}$,

$$
\mathcal{L}_{J \xi} \tau=d \tau(J \xi)=p^{*} \theta(J \xi)=\theta\left(J \theta^{\sharp}\right)=g\left(J \theta^{\sharp}, \theta^{\sharp}\right)=0,
$$

and hence $\psi_{t}^{*} \tau=\tau$ for every $t \in \mathbb{R}$. Using the fact that $\mathcal{L}_{J \theta^{\sharp}} g=0, \mathcal{L}_{J \theta^{\sharp}} \omega=0$. This implies that $\psi_{t}^{*} \Omega=\psi_{t}^{*} e^{-\tau} \psi_{t}^{*} p^{*} \omega=e^{-\tau} p^{*} \hat{\psi}_{t}^{*} \omega=e^{-\tau} p^{*} \omega=\Omega$.

Let $\mathbb{R} \rightarrow \tilde{M} \stackrel{\pi}{\longrightarrow} W$ be the principal bundle where $\mathbb{R}=\left\{\varphi_{t}\right\}_{t \in \mathbb{R}}$ (cf. Lemma 2.2). Define the centralizer of $\mathbb{R}$ in $\mathcal{H}(\tilde{M}, \Omega, J)$ to be:

Definition 2.1. $\mathcal{C}_{\mathcal{H}}(\mathbb{R})=\left\{f \in \mathcal{H}(\tilde{M}, \Omega, J) \mid f \circ \varphi_{t}=\varphi_{t} \circ f\right.$ for $\left.\forall t \in \mathbb{R}\right\}$.

As $\tilde{\mathcal{C}}$ centralizes the fundamental group $\pi_{1}(M)$, noting the remark below (2.1),

$$
\pi_{1}(M) \subset \mathcal{C}_{\mathcal{H}}(\mathbb{R}) .
$$

Lemma 2.7. There exists a homomorphism $\nu: \mathcal{C}_{\mathcal{H}}(\mathbb{R}) \rightarrow \operatorname{PSH}(W, \eta, J)$ for which $\pi: \tilde{M} \rightarrow W$ becomes $\nu$-equivariant. Moreover, there is a splitting homomorphism

$q: \operatorname{PSH}(W, \eta, J) \rightarrow \mathcal{C}_{\mathcal{H}}(\mathbb{R})$.

Proof. By definition, any element $f \in \mathcal{C}_{\mathcal{H}}(\mathbb{R})$ satisfies $f_{*} \xi=\xi$. As $f^{*} \Omega=\rho(f) \Omega$, choosing $e^{s}=\rho(f)$, put $\gamma=\varphi_{-s} \circ f$. Then, $\gamma^{*} \Omega=\Omega$. In particular, $\gamma$ leaves $W$ invariant. Let $\gamma^{\prime}$ be the restriction of $\gamma$ to $W$ (i.e., $i \circ \gamma^{\prime}=\gamma$ ). Using (2.6) and $\gamma_{*} \xi=\xi$, we have that $\gamma^{\prime *} \eta=\gamma^{*} \mathcal{L}_{\xi} \Omega=\mathcal{L}_{\xi} \Omega=\eta$. Hence $\gamma^{\prime} \in \operatorname{PSH}(W, \eta, J)$. If we define $\nu(f)=\gamma^{\prime}$, then it is easy to see that $\nu$ is a well defined homomorphism. Let $x=\varphi_{t} w$ be a point in $\tilde{M}$. As $\pi(x)=w$, $\pi(f x)=\pi\left(\varphi_{s} \gamma\left(\varphi_{t} w\right)\right)=\pi\left(\varphi_{s} \varphi_{t} i \gamma^{\prime} w\right)=\pi\left(i \gamma^{\prime} w\right)=\gamma^{\prime} w=\nu(f) \pi(x)$, so $\pi$ is $\nu$-equivariant.

For $\gamma \in \operatorname{PSH}(W, \eta, J)$, we define a diffeomorphism $\tilde{\gamma}: \tilde{M} \rightarrow \tilde{M}$ to be

$$
\tilde{\gamma}(x)=\tilde{\gamma}\left(\varphi_{t} w\right)=\varphi_{t} \gamma w .
$$

By definition, $\pi \circ \tilde{\gamma}=\gamma \circ \pi$ and the $t$-coordinate satisfies that $\tilde{\gamma}^{*} t=t$. Using (2.15) and $\gamma^{*} \eta=\eta$, it follows that $\tilde{\gamma}^{*} \Omega=d\left(e^{\gamma^{*} t} \pi^{*} \gamma^{*} \eta\right)=d\left(e^{t} \pi^{*} \eta\right)=\Omega$. To see that $\tilde{\gamma}: \tilde{M} \rightarrow \tilde{M}$ is 
holomorphic, notice that $\tilde{\gamma}_{*} \xi=\xi$. As $\tilde{\gamma}\left(\psi_{\theta} x\right)=\tilde{\gamma}\left(\psi_{\theta} \varphi_{t} w\right)=\tilde{\gamma}\left(\varphi_{t} i \psi_{\theta}^{\prime} w\right)=\varphi_{t} i \gamma \psi_{\theta}^{\prime} w$, and $\gamma_{*} A=A$,

$$
\begin{aligned}
& \tilde{\gamma}_{*}\left(-J \xi_{x}\right)=\tilde{\gamma}_{*}\left(\left.\frac{d \psi_{\theta}}{d \theta}(x)\right|_{\theta=0}\right)=\left(\left.\frac{d \varphi_{t} i \gamma\left(\psi_{\theta}^{\prime} w\right)}{d \theta}\right|_{\theta=0}\right) \\
& =\varphi_{t_{*}} i_{*} \gamma_{*}\left(\left.\frac{d \psi^{\prime} \theta}{d \theta}(w)\right|_{\theta=0}\right)=\varphi_{t_{*}} i_{*} \gamma_{*} A_{w}=\varphi_{t_{*}} i_{*} A_{\gamma w}=\varphi_{t_{*}}\left(-J \xi_{\gamma w}\right)=-J \xi_{\tilde{\gamma} x} .
\end{aligned}
$$

Hence, $\tilde{\gamma}$ preserves $\{\xi, J \xi\}^{\perp}$. Since the complex structure $J$ : Null $\eta \rightarrow$ Null $\eta$ is defined by the commutative diagram (2.11), $J \gamma_{*}\left(\pi_{*} X\right)=\gamma_{*} J\left(\pi_{*} X\right)$ for $X \in\{\xi, J \xi\}^{\perp}$ by definition. Then $\pi_{*} \tilde{\gamma}_{*} J(X)=J \gamma_{*} \pi_{*}(X)=J \pi_{*} \tilde{\gamma}_{*}(X)=\pi_{*} J \tilde{\gamma}_{*}(X)$. As a consequence, $\tilde{\gamma}_{*} \circ J=J \circ \tilde{\gamma}_{*}$ on $\tilde{M}$. Hence, $\tilde{\gamma} \in \mathcal{C}_{\mathcal{H}}(\mathbb{R})$. It is easy to check that $q(\gamma)=\tilde{\gamma}$ is a homomorphism of $\operatorname{PSH}(W, \eta, J)$ into $\mathcal{C}_{\mathcal{H}}(\mathbb{R})$ such that $\nu \circ q=$ id.

Remark 2.1. $\quad$ From this lemma, there is an isomorphism $\mathcal{C}_{\mathcal{H}}(\mathbb{R}) \approx \mathbb{R} \times \operatorname{PSH}(W, \eta, J)$ where each element of $\mathcal{C}_{\mathcal{H}}(\mathbb{R})$ is described as $\varphi_{s} \cdot q(\alpha)$ for $s \in \mathbb{R}, \alpha \in \operatorname{PSH}(W, \eta, J)$. It acts on $\tilde{M}$ as

$$
\varphi_{s} \cdot q(\alpha)\left(\varphi_{t} \cdot w\right)=\varphi_{s+t} \cdot \alpha w
$$

for which there is an equivariant principal bundle:

$$
\mathbb{R} \rightarrow\left(\mathcal{C}_{\mathcal{H}}(\mathbb{R}), \tilde{M}\right) \stackrel{(\nu, \pi)}{\longrightarrow}(\mathrm{PSH}(W, \eta, J), W) .
$$

2.3. Central group extension. Consider the exact sequence:

$$
1 \rightarrow \mathbb{R} \rightarrow \mathcal{C}_{\mathcal{H}}(\mathbb{R}) \stackrel{\nu}{\longrightarrow} \operatorname{PSH}(W, \eta, J) \rightarrow 1 .
$$

Suppose that $\mathbb{R} \cap \pi_{1}(M)$ is nontrivial. Then it is an infinite cyclic subgroup $\mathbb{Z}$ such that the quotient group $\mathbb{R} / \mathbb{Z}$ is a circle $S^{1}$. Put $Q=\nu\left(\pi_{1}(M)\right) \subset \operatorname{PSH}(W, \eta, J)$. We have a central group extension:

$$
1 \rightarrow \mathbb{Z} \rightarrow \pi_{1}(M) \stackrel{\nu}{\longrightarrow} Q \rightarrow 1 .
$$

The above principal bundle restricts to the following one:

$$
(\mathbb{Z}, \mathbb{R}) \rightarrow\left(\pi_{1}(M), \tilde{M}\right) \stackrel{(\nu, \pi)}{\longrightarrow}(Q, W) .
$$

As both $\mathbb{R}$ and $\pi_{1}(M)$ act properly on $\tilde{M}, Q$ acts also properly discontinuously (but not necessarily freely) on $W$ such that the quotient Hausdorff space $W / Q$ is compact. Since $\rho(\mathbb{Z}) \subset \rho(\mathbb{R})=\mathbb{R}^{+}$from $\S 2.1, \rho(\mathbb{Z})$ is an infinite cyclic subgroup of $\mathbb{R}^{+}$. We need the following lemma. (Compare [9], [4].)

Lemma 2.8. Let $1 \rightarrow \mathbb{Z} \rightarrow \pi_{1}(M) \stackrel{\nu}{\longrightarrow} Q \rightarrow 1$ be the central extension as in (2.24). Then, $\pi_{1}(M)$ has a splitting subgroup $\pi^{\prime}$ of finite index: $1 \rightarrow \mathbb{Z} \rightarrow \pi^{\prime} \stackrel{\nu}{\longrightarrow} Q^{\prime} \rightarrow 1$ In particular, there exists a subgroup $H^{\prime}$ of $\pi^{\prime}$ which maps isomorphically onto a subgroup $Q^{\prime}$ of finite index in $Q$. 
Proof. Consider the homomorphism $\rho^{\prime}=\left.\rho\right|_{\pi_{1}(M)}: \pi_{1}(M) \longrightarrow \mathbb{R}^{+}$from (2.1). Then, $\rho^{\prime}\left(\pi_{1}(M)\right)$ is a free abelian group of rank $k \geq 1$. If we note that $\rho^{\prime}(\mathbb{Z})$ is an infinite cyclic subgroup of $\rho^{\prime}\left(\pi_{1}(M)\right)$, then we can choose a subgroup $G$ of finite index in $\rho^{\prime}\left(\pi_{1}(M)\right)$ such that $\rho^{\prime}(\mathbb{Z})$ is a direct summand in $G ; G=\rho^{\prime}(\mathbb{Z}) \times \mathbb{Z}^{k-1}$. Put $\pi^{\prime}=\rho^{\prime-1}(G)$ and $H^{\prime}=\rho^{\prime-1}\left(\mathbb{Z}^{k-1}\right)$. Then, $\pi^{\prime}$ has finite index in $\pi_{1}(M)$. Obviously $\nu$ maps $H^{\prime}$ isomorphically onto $\nu\left(H^{\prime}\right)=Q^{\prime}$ which is of finite index in $Q$.

Proposition 2.5. The subgroup $Q^{\prime}$ acts freely on $W$ so that the orbit space $W / Q^{\prime}$ is a closed strictly pseudoconvex pseudo-Hermitian manifold induced from the pseudo-Hermitian structure $(\eta, J)$ on $W$.

Proof. Let $f=\nu^{\prime-1}: Q^{\prime} \rightarrow H^{\prime}$ be the inverse isomorphism. For each $\alpha^{\prime} \in Q^{\prime}$ there exists a unique element $\lambda\left(\alpha^{\prime}\right) \in \mathbb{R}$ such that $f\left(\alpha^{\prime}\right)=\varphi_{\lambda\left(\alpha^{\prime}\right)} \cdot q\left(\alpha^{\prime}\right)$. As we know that $Q$ acts properly discontinuously on $W$ from the remark below (2.25), the stabilizer at each point is finite. Suppose that $\alpha^{\prime} w=w$ for some point $w \in W$. As $\alpha^{\prime} \in Q_{w}, \alpha^{\prime \ell}=1$ for some $\ell$. Since $\varphi_{t}$ is a central element and $q$ is a homomorphism, $1=f\left(\alpha^{\prime \ell}\right)=\varphi_{\ell \lambda\left(\alpha^{\prime}\right)} \cdot q\left(\alpha^{\prime \ell}\right)=\varphi_{\ell \lambda\left(\alpha^{\prime}\right)}$. Thus, $\lambda\left(\alpha^{\prime}\right)=0$, i.e., $f\left(\alpha^{\prime}\right)=q\left(\alpha^{\prime}\right)$. By definition of the action $\left(\pi^{\prime}, \tilde{M}\right), f\left(\alpha^{\prime}\right)\left(\varphi_{t} w\right)=$ $q\left(\alpha^{\prime}\right)\left(\varphi_{t} w\right)=\varphi_{t} \alpha^{\prime} w=\varphi_{t} w$. As $\pi^{\prime}$ acts freely on $\tilde{M}, f\left(\alpha^{\prime}\right)=1$ and so $\alpha^{\prime}=1$. If we note that $Q^{\prime} \subset \operatorname{PSH}(W, \eta, J)$, then $(\eta, J)$ induces a pseudo-Hermitian structure $(\hat{\eta}, J)$ on $W / Q^{\prime}$. Here we use the same notation $J$ to the complex structure on Null $\hat{\eta}$.

\section{EXAMPLES OF L.C.K. MANIFOLDS WITH PARALLEL LEE FORM}

In this section we present an explicit construction for the Hopf manifolds.

Let $S^{2 n-1}=\left\{\left.\left(z_{1}, \ldots, z_{n}\right) \in \mathbb{C}^{n}|| z_{1}\right|^{2}+\cdots+\left|z_{n}\right|^{2}=1\right\}$ be the sphere endowed with its standard contact structure

$$
\eta_{0}=\sum_{j=1}^{n}\left(x_{j} d y_{j}-y_{j} d x_{j}\right), \text { where } z_{j}=x_{j}+\sqrt{-1} y_{j}
$$

Let $J_{0}$ be the restriction of the standard complex structure of $\mathbb{C}^{n}$ to $\mathbb{C}^{n}-\{0\}$. It is known that the group of pseudo-Hermitian transformations, $\operatorname{PSH}\left(S^{2 n-1}, \eta_{0}, J_{0}\right)$ is isomorphic with $\mathrm{U}(n)$ (see [20], for example). We define a 1-parameter subgroup $\left\{\psi_{t}\right\}_{t \in \mathbb{R}} \subset \widehat{\operatorname{PSH}}\left(S^{2 n-1}, \eta_{0}, J_{0}\right)$ by the formula:

$$
\psi_{t}\left(z_{1}, \ldots, z_{n}\right)=\left(e^{\mathrm{i} t a_{1}} z_{1}, \ldots, e^{\mathrm{i} t a_{n}} z_{n}\right)
$$

where $\mathrm{i}=\sqrt{-1}$ and $a_{1}, \ldots, a_{n} \in \mathbb{R}$. The vector field induced by this action is

$$
A=\sum_{j=1}^{n} a_{j}\left(x_{j} \frac{d}{d y_{j}}-y_{j} \frac{d}{d x_{j}}\right)
$$

and satisfies $\eta_{0}(A)=a_{1}\left|z_{1}\right|^{2}+\cdots+a_{n}\left|z_{n}\right|^{2}$. 
Now we require that $\eta_{0}(A)>0$ everywhere on $S^{2 n-1}$. Then the numbers $a_{k}$ must satisfy (up to rearrangement):

$$
0<a_{1} \leq \cdots \leq a_{n}
$$

Define a new contact form $\eta_{A}$ on the sphere by

$$
\eta_{A}=\frac{1}{\sum_{j=1}^{n} a_{j}\left|z_{j}\right|^{2}} \cdot \eta_{0}
$$

The contact distributions of $\eta_{0}$ and $\eta_{A}$ coincide, but the characteristic field of $\eta_{A}$ is $A$ : $\eta_{A}(A)=1, \iota_{A} d \eta_{A}=0$. As $A$ generates the flow $\left\{\psi_{t}\right\}_{t \in \mathbb{R}} \subset \operatorname{PSH}\left(S^{2 n-1}, \eta_{0}, J_{0}\right)$, note that $\psi_{t *} \circ J_{0}=J_{0} \circ \psi_{t *}$ on Null $\eta_{A}$. Define a 2 -form on the product $\mathbb{R} \times S^{2 n-1}$ by:

$$
\Omega_{A}=2 d\left(e^{t} \operatorname{pr}^{*} \eta_{A}\right), \quad(t \in \mathbb{R}) .
$$

Here pr : $\mathbb{R} \times S^{2 n-1} \rightarrow S^{2 n-1}$ is the projection. If $\mathbb{R}=\left\{\varphi_{s}\right\}_{s \in \mathbb{R}}$ acts on $\mathbb{R} \times S^{2 n-1}$ by left translations: $\varphi_{s}(t, z)=(s+t, w)$, then the group $\mathbb{R} \times \operatorname{PSH}\left(S^{2 n-1}, \eta_{A}, J_{0}\right)$ acts by homothetic transformations w.r.t. $\Omega_{A}$ :

$$
\left(\varphi_{s} \times \alpha\right)^{*} \Omega_{A}=e^{s} \cdot \Omega_{A}, \quad\left(\alpha \in \operatorname{PSH}\left(S^{2 n-1}, \eta_{A}, J_{0}\right)\right) .
$$

In general, $\operatorname{PSH}\left(S^{2 n-1}, \eta_{A}, J_{0}\right)$ is the centralizer of $\left\{\psi_{t}\right\}_{t \in \mathbb{R}}$ in $\mathrm{U}(n)$. In view of the formula of $\psi_{t}, \operatorname{PSH}\left(S^{2 n-1}, \eta_{A}, J_{0}\right)$ contains the maximal torus of $\mathrm{U}(n)$ at least.

$$
T^{n} \subset \operatorname{PSH}\left(S^{2 n-1}, \eta_{A}, J_{0}\right) \text {. }
$$

(For example, if all $a_{j}$ are distinct, $\left.\operatorname{PSH}\left(S^{2 n-1}, \eta_{0}, J_{0}\right)=T^{n}\right)$.

Let $N=\frac{d}{d t}$ be the vector field induced on $\mathbb{R} \times S^{2 n-1}$ by the $\mathbb{R}$-action. Taking into account that $T\left(\mathbb{R} \times S^{2 n-1}\right)=N \oplus A \oplus$ Null $\eta_{A}$, we define an almost complex structure $J_{A}$ on $\mathbb{R} \times S^{2 n-1}$ by:

$$
\begin{aligned}
& J_{A} N=-A, \quad J_{A} A=N, \\
& J_{A} \mid \text { Null } \eta_{A}=J_{0}
\end{aligned}
$$

and show its integrability. Indeed, let

$$
T\left(\mathbb{R} \times S^{2 n-1}\right) \otimes \mathbb{C}=\left\{T^{1,0}+(A-\mathrm{i} N)\right\} \oplus\left\{T^{0,1}+(A+\mathrm{i} N)\right\}
$$

be the splitting corresponding to $J_{A}$ (here $T^{1,0}+T^{0,1}=\operatorname{Null} \eta_{A} \otimes \mathbb{C}$ ). As $J_{A} \mid$ Null $\eta_{A}=J_{0}$, $\left[T^{1,0}, T^{0,1}\right] \subset T^{1,0}$. Recalling that $A$ is the characteristic field of $\eta_{A}$, we see that $[X, A] \in \operatorname{Null} \eta_{A}$ for any $X \in \operatorname{Null} \eta_{A}$. If $X \in T^{1,0}$, then $[X, A-\mathrm{i} N]=[X, A]=$ $\lim _{t \rightarrow 0} \frac{X-\psi_{-t *} X}{t}$. Noting that $\psi_{t} \in \operatorname{PSH}\left(S^{2 n-1}, \eta_{A}, J_{0}\right)$ (i.e., $\left.\psi_{t *} J_{0}=J_{0} \psi_{t *}\right)$,

$$
\begin{aligned}
J_{A}[X, A-\mathrm{i} N] & =J_{0}[X, A]=\lim _{t \rightarrow 0} \frac{J_{0} X-\psi_{-t *} J_{0} X}{t}=\left[J_{0} X, A\right] \\
& =[\mathrm{i} X, A]=\mathrm{i}[X, A]=\mathrm{i}[X, A-\mathrm{i} N] .
\end{aligned}
$$

Thus $[X, A-\mathrm{i} N] \in\left\{T^{1,0}+(A-\mathrm{i} N)\right\}$. Hence $J_{A}$ is integrable. By the definition of $J_{A}$, it is easy to check that the elements of $\mathbb{R} \times \operatorname{PSH}\left(S^{2 n-1}, \eta_{A}, J_{0}\right)$ are holomorphic w.r.t. $J_{A}$. Moreover, $\Omega_{A}$ is $J_{A}$-invariant. Hence, $\Omega_{A}$ is a Kähler form on the complex manifold ( $\mathbb{R} \times$ 
$\left.S^{2 n-1}, J_{A}\right)$ on which $\mathbb{R} \times \operatorname{PSH}\left(S^{2 n-1}, \eta_{A}, J_{0}\right)$ acts as the group of holomorphic homothetic transformations. Define a Hermitian metric $\tilde{g}_{A}$ and its fundamental 2-form $\tilde{\omega}_{A}$ by setting

$$
\begin{aligned}
& \tilde{\omega}_{A}=2 e^{-t} \cdot \Omega_{A} . \\
& \tilde{g}_{A}(X, Y)=\tilde{\omega}_{A}\left(J_{A} X, Y\right), \quad \forall X, Y \in T\left(\mathbb{R} \times S^{2 n-1}\right) .
\end{aligned}
$$

(Compare (2.16).) By (3.3), $\mathbb{R} \times \operatorname{PSH}\left(S^{2 n-1}, \eta_{A}, J_{0}\right)$ acts as holomorphic isometries of $\left(\tilde{g}_{A}, J_{A}\right)$. When we choose a properly discontinuous group $\Gamma \subset \mathbb{R} \times \operatorname{PSH}\left(S^{2 n-1}, \eta_{A}, J_{0}\right)$ acting freely on $\mathbb{R} \times S^{2 n-1}, \tilde{g}_{A}$ (resp. $\tilde{\omega}_{A}$ ) induces a Hermitian metric $g_{A}$ (resp. the fundamental 2-form $\left.\omega_{A}\right)$ on the quotient complex manifold $\left(\mathbb{R} \times S^{2 n-1} / \Gamma, \hat{J}_{A}\right)$, where the complex structure $\hat{J}_{A}$ is induced from $J_{A}$. We have to check that $g_{A}$ is a l.c.K. metric with parallel Lee form. Let $p: \mathbb{R} \times S^{2 n-1} \rightarrow \mathbb{R} \times S^{2 n-1} / \Gamma$ be the projection so that $p^{*} \omega_{A}=\tilde{\omega}_{A}$. Since $\tilde{\omega}_{A}=e^{-t} \cdot \Omega_{A}$, we have $d \tilde{\omega}_{A}=-d t \wedge \tilde{\omega}_{A}$. Thus $\tilde{g}_{A}$ is a l.c.K. metric with Lee form $d(-t)$ on $\mathbb{R} \times S^{2 n-1}$. If we note that the group $\mathbb{R} \times \operatorname{PSH}\left(S^{2 n-1}, \eta_{A}, J_{0}\right)$ leaves $d(-t)$ invariant, i.e. $\left(\varphi_{s} \times \alpha\right)^{*} d(-t)=d(-(s+t))=d(-t)$, then $d(-t)$ induces a 1-form $\theta$ on $\mathbb{R} \times S^{2 n-1} / \Gamma$ such that $p^{*} \theta=d(-t)$. The equation $d \tilde{\omega}_{A}=-d t \wedge \tilde{\omega}_{A}$ implies that $d \omega_{A}=\theta \wedge \omega_{A}$ on $\mathbb{R} \times S^{2 n-1} / \Gamma$. As $d \theta=0, g_{A}$ is a l.c.K. metric with Lee form $\theta$. For the rest, the same argument as in the proof of Lemma 2.5 can be applied to show that $\theta$ is the parallel Lee form of $g_{A}$. Finally, we examine the complex structure $\hat{J}_{A}$ on $\mathbb{R} \times S^{2 n-1} / \Gamma$. Let $H: \mathbb{R} \times S^{2 n-1} \rightarrow \mathbb{C}^{n}-\{0\}$ be the diffeomorphism defined by:

$$
H\left(t,\left(z_{1}, \ldots, z_{n}\right)\right)=\left(e^{-a_{1} t} z_{1}, \ldots, e^{-a_{n} t} z_{n}\right),
$$

where $\left\{a_{1}, \ldots, a_{n}\right\}$ satisfies the condition (3.2). We shall show that $H$ is a $\left(J_{A}, J_{0}\right)$ biholomorphism. We have:

$$
\begin{aligned}
H_{*}\left(N_{(s, z)}\right) & =\left.\frac{d H(t+s, z)}{d t}\right|_{t=0}=\left(-a_{1} \cdot e^{-a_{1} s} \cdot z_{1}, \ldots,-a_{n} \cdot e^{-a_{n} s} \cdot z_{n}\right) ; \\
H_{*}\left(J_{A} N_{(s, z)}\right) & =H_{*}\left(-A_{(s, z)}\right)=-H_{*}\left(\left(s,\left.\frac{d}{d t}\left(e^{\mathrm{i} t a_{1}} z_{1}, \ldots, e^{\mathrm{i} t a_{n}} z_{n}\right)\right|_{t=0}\right)\right. \\
& =-\left(\mathrm{i} a_{1} e^{-a_{1} s} z_{1}, \ldots, \mathrm{i} a_{n} e^{-a_{n} s} z_{n}\right)=J_{0} H_{*}\left(N_{(s, z)}\right) .
\end{aligned}
$$

From $H_{*}\left(A_{(s, z)}\right)=-J_{0} H_{*}\left(N_{(s, z)}\right)$, we derive $J_{0} H_{*}\left(A_{(s, z)}\right)=H_{*}\left(N_{(s, z)}\right)=H_{*}\left(J_{A} A\right)$. Now let $X \in$ Null $\eta_{A} \subset T S^{2 n-1}$ and let $\sigma(t)$ be an integral curve of $X$ on $S^{2 n-1}: \dot{\sigma}(t)=X$, $\dot{\sigma}(0)=X_{z}$. We can view $X$ as a pair: $X_{(s, z)}=(s, \dot{\sigma}(0))$. Then:

$$
H_{*}\left(X_{(s, z)}\right)=\left.\frac{d}{d t} H(s, \sigma(t))\right|_{t=0}=\left(e^{-a_{1} s} \dot{\sigma}_{1}(0), \ldots, e^{-a_{n} s} \dot{\sigma}_{n}(0)\right) .
$$

From this we obtain:

$$
\begin{aligned}
H_{*}\left(J_{A} X_{(s, z)}\right) & =H_{*}\left(\left(s, J_{0} \dot{\sigma}(0)\right)\right)=H_{*}\left(\left(s,\left(\mathrm{i} \dot{\sigma}_{1}(0), \ldots, \mathrm{i} \dot{\sigma}_{n}(0)\right)\right)\right) \\
& =\left(\mathrm{i} e^{-a_{1} s} \dot{\sigma}_{1}(0), \ldots, \mathrm{i} e^{-a_{n} s} \dot{\sigma}_{n}(0)\right) \\
& =J_{0}\left(e^{-a_{1} s} \dot{\sigma}_{1}(0), \ldots, e^{-a_{n} s} \dot{\sigma}_{n}(0)\right)=J_{0} H_{*}\left(X_{(s, z)}\right) .
\end{aligned}
$$

Therefore $H:\left(\mathbb{R} \times S^{2 n-1}, J_{A}\right) \rightarrow\left(\mathbb{C}^{n}-\{0\}, J_{0}\right)$ is a biholomorphism.

Let $\operatorname{Hol}\left(\mathbb{C}^{n}-\{0\}, J_{0}\right)$ be the group of all biholomorphic transformations. If we associate to each $\gamma \in \mathbb{R} \times \operatorname{PSH}\left(S^{2 n-1}, \eta_{A}, J_{0}\right)$ the biholomorphic map $H \circ \gamma \circ H^{-1}$, we obtain a faithful 
homomorphism $\mathbb{R} \times \operatorname{PSH}\left(S^{2 n-1}, \eta_{A}, J_{0}\right) \longrightarrow \operatorname{Hol}\left(\mathbb{C}^{n}-\{0\}, J_{0}\right)$. Let $\Gamma_{H}$ be the image of $\Gamma$ in $\operatorname{Hol}\left(\mathbb{C}^{n}-\{0\}, J_{0}\right)$.

Definition 3.1. The quotient complex manifold $\mathbb{C}^{n}-\{0\} / \Gamma_{H}$ is called a Hopf manifold.

We have shown:

Theorem 3.1. The Hopf manifold $\mathbb{C}^{n}-\{0\} / \Gamma_{H}$ admits a l.c.K. metric $g$ with parallel Lee form $\theta$.

By (3.4), $T^{n} \subset \operatorname{PSH}\left(S^{2 n-1}, \eta_{A}, J_{0}\right)$. Choose $s \in \mathbb{R}-\{0\}$ and $n$-complex numbers $c_{1}, \ldots, c_{n} \in S^{1}$. Consider an infinite cyclic subgroup $\mathbb{Z}$ generated by the element

$\left(s,\left(c_{1}, \ldots, c_{n}\right)\right)$ from $\mathbb{R} \times \operatorname{PSH}\left(S^{2 n-1}, \eta_{0}, J_{0}\right)$. Then the corresponding group $\mathbb{Z}_{H}$ is generated by the element $\left(e^{-a_{1} s} \cdot c_{1}, \ldots, e^{-a_{n} s} \cdot c_{n}\right)$ acting on $\mathbb{C}^{n}-\{0\}$. Let $\Lambda=\left(\lambda_{1}, \ldots, \lambda_{n}\right)$, with $\lambda_{j}=e^{-a_{j} s} \cdot c_{j}$ and so $\mathbb{Z}_{H}=<\left(\lambda_{1}, \ldots, \lambda_{n}\right)>$. The condition (3.2) ensures that the complex numbers $\lambda_{j}$ satisfy

$$
0<\left|\lambda_{n}\right| \leq \cdots \leq\left|\lambda_{1}\right|<1
$$

Put $M_{\Lambda}=\mathbb{C}^{n}-\{0\} / \Gamma_{H}$. We call $M_{\Lambda}$ a primary Hopf manifold of type $\Lambda$. Indeed, for $n=2$, one recovers the primary Hopf surfaces of Kähler rank 1. In particular, we derive Theorem B in the Introduction.

Remark 3.1. Note that the manifolds $M_{\Lambda}$ are all diffeomorphic with $S^{1} \times S^{2 n-1}$ and that for $c_{1}=\cdots=c_{n}=1$ and $a_{1}=\cdots=a_{n}$, we obtain the standard Hopf manifold, the first known example of a l.c.K. manifold with parallel Lee form, cf. [17].

In [6] a l.c.K. metric with parallel Lee form is constructed on the primary Hopf surface $M_{\lambda_{1}, \lambda_{2}}=\mathbb{C}^{2}-\{0\} / \Gamma, \Gamma \cong \mathbb{Z}$ generated by $\left(z_{1}, z_{2}\right) \mapsto\left(\lambda_{1} z_{1}, \lambda_{2} z_{2}\right),\left|\lambda_{1}\right| \geq\left|\lambda_{2}\right|>1$. There the diffeomorphism between $M_{\lambda_{1}, \lambda_{2}}$ and $S^{1} \times S^{3}$ is used to construct a potential for the Kähler metric $h$ (in the present paper notations) on the universal cover. The same diffeomorphism is then used to transport the l.c.K. structure on $S^{1} \times S^{3}$ and to show that the induced Sasakian structure on $S^{3}$ is a deformation of the standard Sasakian structure of the 3sphere. See also [1] where a complete list of compact, complex surfaces admitting l.c.K. metrics with parallel Lee form is provided.

\section{LeE-CAUChy-RiEmann transformations}

In this section, we consider the group $\operatorname{Aut}_{L C R}(M)$ described in the Introduction. Let $\left\{\theta, \theta \circ J, \theta^{\alpha}, \bar{\theta}^{\alpha}\right\}_{\alpha=1, \cdots, n-1}$ be a unitary, local coframe field adapted to a l.c.K. manifold $(M, g, J)$. Consider the subgroup $G$ of $\mathrm{GL}(2 n, \mathbb{R})$ consisting of the following elements:

$$
\left\{\left(\begin{array}{cccc}
1 & 0 & 0 & 0 \\
0 & u & v^{\alpha} & \bar{v}^{\alpha} \\
0 & 0 & \sqrt{u} U_{\beta}^{\alpha} & 0 \\
0 & 0 & 0 & \sqrt{u} \bar{U}_{\beta}^{\alpha}
\end{array}\right) \mid u \in \mathbb{R}^{+}, v^{\alpha} \in \mathbb{C}, U_{\beta}^{\alpha} \in \mathrm{U}(n-1)\right\} .
$$

Let $G \rightarrow P \rightarrow M$ be the principal bundle of the $G$-structure consisting of the above coframes $\left\{\theta, \theta \circ J, \theta^{\alpha}, \bar{\theta}^{\alpha}\right\}$. If we note that $G$ is isomorphic to the semidirect product $\mathbb{C}^{n-1} \rtimes\left(\mathrm{U}(n-1) \times \mathbb{R}^{+}\right)$, then the Lie algebra $\mathfrak{g}$ is isomorphic to $\mathbb{C}^{n-1}+\mathfrak{u}(n-1)+\mathbb{R}$. In particular, the matrix group $\mathfrak{g} \subset \mathfrak{g l}(2 n, \mathbb{R})$ has no element of rank 1 , i.e. it is elliptic (cf. 
[10]). Note that $\mathbb{C}^{n-1}$ is of infinite type, while $\mathfrak{u}(n-1)+\mathbb{R}$ is of order 2 . As $M$ is assumed to be compact, the group of automorphisms $\mathcal{U}$ of $P$ is a (finite dimensional) Lie group.

Definition 4.1. The group of all diffeomorphisms of $M$ onto itself which preserve the above $G$-structure is denoted by $\operatorname{Aut}_{L C R}(M, g, J, \theta)$ (or simply by $\operatorname{Aut}_{L C R}(M)$ ). We call $\operatorname{Aut}_{L C R}(M)$ the group of Lee-Cauchy-Riemann transformations on a l.c.K. manifold $(M, g, J)$ adapted to the Lee form $\theta$.

By definition, if $f \in \operatorname{Aut}_{L C R}(M)$, then $f^{*}: P \rightarrow P$ is a bundle automorphism satisfying

$$
\begin{aligned}
& f^{*} \theta=\theta, \\
& f^{*}(\theta \circ J)=\lambda \cdot(\theta \circ J), \text { for some positive, smooth function } \lambda, \\
& f^{*} \theta^{\alpha}=\sqrt{\lambda} \cdot \theta^{\beta} V_{\beta}^{\alpha}+(\theta \circ J) \cdot w^{\alpha}, \\
& f^{*} \bar{\theta}^{\alpha}=\sqrt{\lambda} \cdot \bar{\theta}^{\beta} \bar{V}_{\beta}^{\alpha}+(\theta \circ J) \cdot \bar{w}^{\alpha},
\end{aligned}
$$

for functions $V_{\beta}^{\alpha} \in \mathrm{U}(n-1)$ and $w^{\alpha} \in \mathbb{C}$. Note that the group of holomorphic isometries $\mathrm{I}(M, g, J)$ is contained in $\operatorname{Aut}_{L C R}(M)$. In fact, an element $f \in \mathrm{I}(M, g, J)$ satisfies $f^{*} \theta=$ $\theta, f^{*}(\theta \circ J)=(\theta \circ J)$ and $f^{*} \omega=\omega$. Let $\left\{\theta^{\sharp}, J \theta^{\sharp}\right\}^{\perp}$ be the orthogonal complement of the complex plane field $\left\{\theta^{\sharp}, J \theta^{\sharp}\right\}$ w.r.t. $g$. It is obviously $J$-invariant. If we note that $\omega \mid\left\{\theta^{\sharp}, J \theta^{\sharp}\right\}^{\perp}=-\mathrm{i} \sum_{\alpha, \beta} \delta_{\alpha \beta} \theta^{\alpha} \wedge \bar{\theta}^{\beta}$, then $f^{*} \theta^{\alpha}=\theta^{\beta} U_{\beta}^{\alpha}, f^{*} \bar{\theta}^{\alpha}=\bar{\theta}^{\beta} \bar{U}_{\beta}^{\alpha}$ for some matrix function $U_{\beta}^{\alpha} \in \mathrm{U}(n-1)$.

Lemma 4.1. Any element $f \in \operatorname{Aut}_{L C R}(M)$ preserves $\left\{\theta^{\sharp}, J \theta^{\sharp}\right\}^{\perp}$ and is holomorphic on it.

Proof. Let $X \in\left\{\theta^{\sharp}, J \theta^{\sharp}\right\}^{\perp}$. The equations $f^{*} \theta=\theta, f^{*}(\theta \circ J)=\lambda \cdot(\theta \circ J)$ show that

$$
\begin{aligned}
g\left(f_{*} X, \theta^{\sharp}\right) & =\theta\left(f_{*} X\right)=\theta(X)=g\left(X, \theta^{\sharp}\right)=0, \\
g\left(f_{*} X, J \theta^{\sharp}\right) & =-g\left(J f_{*} X, \theta^{\sharp}\right)=-\theta\left(J f_{*} X\right)=-\theta \circ J\left(f_{*} X\right) \\
& =-\lambda \cdot \theta \circ J(X)=-g\left(X,(\theta \circ J)^{\sharp}\right)=g\left(X, J \theta^{\sharp}\right)=0 .
\end{aligned}
$$

Thus $f_{*}$ applies $\left\{\theta^{\sharp}, J \theta^{\sharp}\right\}^{\perp}$ onto itself. Moreover, if $\theta_{\alpha}^{\sharp}$ is a dual frame field to $\theta^{\alpha}$ (similarly for $\left.\bar{\theta}^{\alpha}\right)$, then the frame $\left\{\theta_{\alpha}^{\sharp}, \bar{\theta}_{\alpha}^{\sharp}\right\}_{\alpha=1, \cdots, n-1}$ spans $\left\{\theta^{\sharp}, J \theta^{\sharp}\right\}^{\perp} \otimes \mathbb{C}$.

The equation $f^{*} \theta^{\alpha}=\sqrt{\lambda} \cdot \theta^{\beta} V_{\beta}^{\alpha}+(\theta \circ J) \cdot w^{\alpha}$ implies that $f_{*} \theta_{\alpha}^{\sharp}=\sqrt{\lambda} \cdot \theta_{\beta}^{\sharp} V_{\alpha}^{\beta} \quad$ (similary for $f_{*} \bar{\theta}_{\alpha}^{\sharp}$. Therefore $f_{*} \circ J=J \circ f_{*}$ on $\left\{\theta^{\sharp}, J \theta^{\sharp}\right\}^{\perp}$.

When a noncompact $L C R$ flow exists on a compact l.c.K. manifold $M$ with parallel Lee form, we shall prove a rigidity similar to the one implied by a noncompact $C R$-flow on a compact $C R$-manifold (cf. [14], [8]).

\section{Proof of Theorem C.}

4.1. Existence of spherical $C R$-structure on $W / Q^{\prime}$. Let $1 \rightarrow \mathbb{Z} \rightarrow \pi^{\prime} \stackrel{\nu}{\longrightarrow} Q^{\prime} \rightarrow 1$ be the split central group extension from Lemma 2.8. Put $M^{\prime}=\tilde{M} / \pi^{\prime}$. Then it is easy to see that the Lee form $\theta$, the LCR-action $\mathbb{C}^{*}$ lift to those of $M^{\prime}$, so we retain the same notations for $M^{\prime}$. We put $\mathbb{C}^{*}=S^{1} \times \mathbb{R}^{+}$where $\mathbb{R}^{+}=\left\{\hat{\phi}_{t}\right\}_{t \in \mathbb{R}}$ is a $L C R$ flow on $M^{\prime}$. By hypothesis, $S^{1}=\left\{\hat{\varphi}_{t}\right\}_{t \in \mathbb{R}}$ induces the Lee field $\theta^{\sharp}$. From 1 of Proposition 2.4, $S^{1}$ lifts to a nontrivial 
holomorphic homothetic flow $\mathbb{R}=\left\{\varphi_{t}\right\}_{t \in \mathbb{R}}$ on $\tilde{M}$ w.r.t. $\Omega$. We obtain a LCR-action of $\mathbb{R} \times \mathbb{R}^{+}$on $\tilde{M}$ for which $\mathbb{R}$ acts properly as before. Consider the commutative diagram of principal bundles:

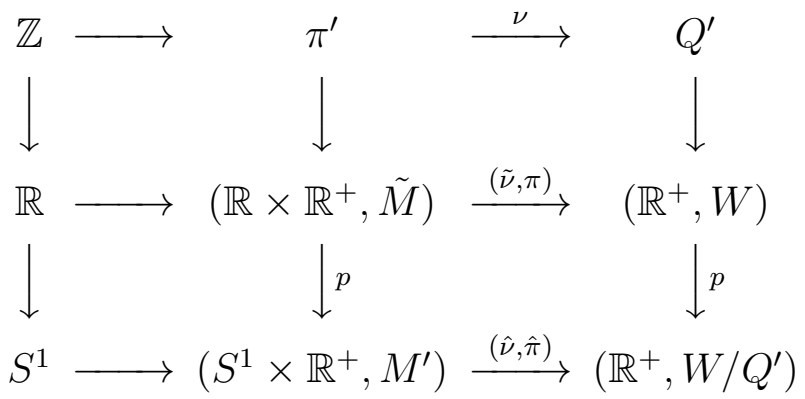

From the bottom line, the projection $\hat{\nu}$ maps the group $\mathbb{R}^{+}=\left\{\hat{\phi}_{t}\right\}_{t \in \mathbb{R}}$ onto a group $\mathbb{R}^{+}=$ $\left\{\bar{\phi}_{t}\right\}_{t \in \mathbb{R}}$ acting on $W / Q^{\prime}$.

Lemma 4.2. The group $\mathbb{R}^{+}=\left\{\bar{\phi}_{t}\right\}_{t \in \mathbb{R}}$ acts by $C R$-transformations on $W / Q^{\prime}$ w.r.t. the $C R$-structure induced from the strictly pseudoconvex, pseudo-Hermitian structure $(\hat{\eta}, J)$.

Proof. As $\xi$ generates the flow $\mathbb{R}=\left\{\varphi_{t}\right\}_{t \in \mathbb{R}}, p_{*} \xi=\theta^{\sharp}$ on $M^{\prime}$ by hypothesis and so $p: \tilde{M} \rightarrow M^{\prime}$ maps the complex plane field $\{\xi, J \xi\}$ onto $\left\{\theta^{\sharp}, J \theta^{\sharp}\right\}$. By Lemma 4.1, each $\hat{\phi}_{t} \in \operatorname{Aut}_{L C R}\left(M^{\prime}\right)$ preserves $\left\{\theta^{\sharp},(\theta \circ J)^{\sharp}\right\}^{\perp}$. So its lift $\phi_{t}$ preserves the $J$-invariant distribution $\{\xi, J \xi\}^{\perp}$. Since $\pi_{*}:\left(\{\xi, J \xi\}^{\perp}, J\right) \rightarrow($ Null $\eta, J)$ is $J$-isomorphic and each $\phi_{t}$ is holomorphic on $\{\xi, J \xi\}^{\perp}, \hat{\pi}_{*}:\left(\left\{\theta^{\sharp},(\theta \circ J)^{\sharp}\right\}^{\perp}, J\right) \longrightarrow($ Null $\hat{\eta}, J)$ is also $J$-isomorphic through the commutative diagram and thus each $\bar{\phi}_{t}$ is holomorphic on Null $\hat{\eta} ;\left(\bar{\phi}_{t *} \circ J=J \circ \bar{\phi}_{t *}\right)$. Therefore, $\mathbb{R}^{+}=\left\{\bar{\phi}_{t}\right\}_{t \in \mathbb{R}}$ is a closed, noncompact subgroup of $C R$-transformations of $W / Q^{\prime}$ w.r.t. (Null $\hat{\eta}, J)$.

By this lemma, we obtain a compact strictly pseudoconvex $C R$-manifold $W / Q^{\prime}$ admitting a closed, noncompact $C R$-transformations $\mathbb{R}^{+}$. Then we apply the result of $\mathbb{8}$ to show that $W / Q^{\prime}$ is $C R$-equivalent to the sphere $S^{2 n-1}$ with the standard $C R$-structure. In particular $Q^{\prime}=\{1\}$ and thus $Q$ is a finite subgroup of $\operatorname{PSH}(W, \eta, J)$ from Lemma 2.8. By definition of spherical $C R$-structure (cf. [12], [7]), there exists a developing pair:

$$
(\mu, \operatorname{dev}):\left(\operatorname{Aut}_{C R}(W), W\right) \rightarrow\left(\mathrm{PU}(n, 1), S^{2 n-1}\right)
$$

for which dev is a $C R$-diffeomorphism and $\mu: \operatorname{Aut}_{C R}(W) \rightarrow \mathrm{PU}(n, 1)$ is the holonomy isomorphism. Here $\mathrm{PU}(n, 1)=\operatorname{Aut}_{C R}\left(S^{2 n-1}\right)$ and $\operatorname{Aut}_{C R}(W)$ is the group of all $C R$ automorphisms of $W$ containing the groups $\mathbb{R}^{+}$and $\operatorname{PSH}(W, \eta, J) \supset Q$.

As $S^{1}\left(\subset \mathbb{C}^{*}\right)$ acts on $M$ without fixed points (but not necessarily freely), the quotient space $M / S^{1}=W / Q\left(\approx S^{2 n-1} / \mu(Q)\right)$ is an orbifold, so such a finite subgroup $Q$ may exist.

On the other hand, we recall some facts from the theory of hyperbolic groups (cf. [3]). The noncompact closed $\mu\left(\mathbb{R}^{+}\right)$-action on $S^{2 n-1}$ is characterized as whether it is either loxodromic $\left(=\mathbb{R}^{+}\right)$or parabolic $(=\mathcal{R})$ for which $\mathbb{R}^{+}$has exactly two fixed points $\{0, \infty\}$ or $\mathcal{R}$ has the unique fixed point $\{\infty\}$ on $S^{2 n-1}$. Moreover, the centralizer $\mathcal{C}_{\mathrm{PU}(n, 1)}\left(\mu\left(\mathbb{R}^{+}\right)\right)$ of $\mu\left(\mathbb{R}^{+}\right)$in $\mathrm{PU}(n, 1)$ is one of the following groups up to conjugacy: 


$$
\mathcal{R} \times \mathrm{U}(n-1) \text { or } \mathbb{R}^{+} \times \mathrm{U}(n-1) .
$$

Since $\pi_{1}(M)$ centralizes $\mathbb{R} \times \mathbb{R}^{+}$, note that $Q$ centralizes $\mathbb{R}^{+}$(cf. (2.24)). The holonomy group $\mu(Q)$ belongs to $\mathcal{C}_{\mathrm{PU}(n, 1)}\left(\mu\left(\mathbb{R}^{+}\right)\right)$. As $\mu(Q)$ is a finite subgroup, (4.4) implies that

$$
\mu(Q) \subset \mathrm{U}(n-1) .
$$

4.2. Rigidity of $(M, g, J)$ under the $L C R$ action of $\mathbb{R}^{+}$. Let $\left(\eta_{0}, J_{0}\right)$ be the standard strictly pseudoconvex pseudo-Hermitian structure on $S^{2 n-1}$ (cf. (3.1)). By definition, there exists a positive function $u$ on $W$ such that

$$
\operatorname{dev}^{*} \eta_{0}=u \cdot \eta .
$$

By Lemma 2.4, we know that $A$ is the characteristic $C R$-vector field on $W$ for $(\eta, J)$. If $\left\{\psi_{t}^{\prime}\right\}$ is the flow generated by $A$, then note from (2.13) that $\left\{\psi_{t}^{\prime}\right\} \subset \operatorname{PSH}(W, \eta, J)$. Because $W$ is compact, $\operatorname{PSH}(W, \eta, J)$ is compact. $\operatorname{As} \operatorname{PSH}(W, \eta, J) \subset \operatorname{Aut}_{C R}(W)$, the closure of the holonomy image $\mu\left(\left\{\psi_{t}^{\prime}\right\}\right)$ (which is a connected abelian group) lies in the maximal torus $T^{n}$ of the maximal compact subgroup $\mathrm{U}(n)$ in $\mathrm{PU}(n, 1)$ up to conjugacy. We can describe it as

$$
\mu\left(\psi_{t}^{\prime}\right)=\left(e^{i a_{1} \cdot t}, \cdots, e^{i a_{n} \cdot t}\right) \quad(\forall t \in \mathbb{R})
$$

for some $a_{i} \in \mathbb{R}(i=1, \ldots, n)$. On the other hand, let $\mathcal{A}=\operatorname{dev}_{*}(A)$. Since $\operatorname{dev}$ is equivariant, $\operatorname{dev}\left(\psi_{t}^{\prime} w\right)=\mu\left(\psi_{t}^{\prime}\right) \operatorname{dev}(w)$ on $S^{2 n-1}=\left\{z=\left.\left(z_{1}, z_{2}, \cdots, z_{n}\right) \in \mathbb{C}^{n}|| z_{1}\right|^{2}+\left|z_{2}\right|^{2}+\cdots+\left|z_{n}\right|^{2}=1\right\}$, we have:

$$
\mathcal{A}_{z}=\frac{d \mu\left(\psi_{t}^{\prime}\right)}{d t}=\sum_{j=1}^{n} a_{j}\left(x_{j} \frac{d}{d y_{j}}-y_{j} \frac{d}{d x_{j}}\right) \quad\left(z=\operatorname{dev}(w), \quad z_{j}=x_{j}+\mathrm{i} y_{j}\right) .
$$

As $\eta(A)=1$, we have

$$
u(w)=\operatorname{dev}^{*} \eta_{0}(A)=\eta_{0}\left(\mathcal{A}_{z}\right)=\sum_{j=1}^{n} a_{j} \cdot\left|z_{j}\right|^{2} .
$$

Since $u>0$ from (4.6), we can assume that

$$
0<a_{1} \leq \cdots \leq a_{n} .
$$

As $\operatorname{dev}^{-1}$ maps the pseudo-Hermitain structure $(\eta, J)$ on $W$ to $\left(\operatorname{dev}^{-1 *} \eta, J_{0}\right)$ on $S^{2 n-1}$, we put

$$
\eta_{\mathcal{A}}=\operatorname{dev}^{-1 *} \eta
$$

Using (4.8), we obtain:

$$
\eta_{\mathcal{A}}=\frac{1}{\sum_{j=1}^{n} a_{j} \cdot\left|z_{j}\right|^{2}} \cdot \eta_{0} \text { on } S^{2 n-1}
$$


When we note that $\eta_{0}=u^{\prime} \cdot \eta_{\mathcal{A}}$ where $u^{\prime}=u \circ \mathrm{dev}^{-1}$, and $T\left(\mathbb{R} \times S^{2 n-1}\right)=\left\{\frac{d}{d t}, \mathcal{A}\right\} \oplus$ Null $\eta_{0}$, denote the complex structure $J_{\mathcal{A}}$ on $\mathbb{R} \times S^{2 n-1}$ by

$$
\begin{aligned}
& J_{\mathcal{A}} \frac{d}{d t}=-\mathcal{A}, \quad J_{\mathcal{A}} \mathcal{A}=\frac{d}{d t} \\
& J_{\mathcal{A}} \mid \text { Null } \eta_{0}=J_{0} .
\end{aligned}
$$

(Compare §3.) Let $\operatorname{Pr}: \mathbb{R} \times S^{2 n-1} \rightarrow S^{2 n-1}$ be the canonical projection. In view of (3.5), setting

$$
\begin{aligned}
& \Omega_{\mathcal{A}}=d\left(e^{t} \cdot \operatorname{Pr}^{*} \eta_{\mathcal{A}}\right), \quad \tilde{\omega}_{\mathcal{A}}=2 e^{-t} \cdot \Omega_{\mathcal{A}}, \\
& \tilde{g}_{\mathcal{A}}(X, Y)=\tilde{\omega}_{\mathcal{A}}\left(J_{\mathcal{A}} X, Y\right),
\end{aligned}
$$

we obtain a l.c.K. structure $\left(\Omega_{\mathcal{A}}, J_{\mathcal{A}}\right)$ on $\mathbb{R} \times S^{2 n-1}$ endowed with the group $\mathbb{R} \times \operatorname{PSH}\left(S^{2 n-1}, \eta_{\mathcal{A}}, J_{0}\right)$ of holomorphic homothetic transformations.

Proposition 4.1. There exists an equivariant holomorphic isometry between $\left(\mathcal{C}_{\mathcal{H}}(\mathbb{R}), \tilde{M}, \Omega, J\right)$ and $\left(\mathbb{R} \times \operatorname{PSH}\left(S^{2 n-1}, \eta_{\mathcal{A}}, J_{0}\right), \mathbb{R} \times S^{2 n-1}, \Omega_{\mathcal{A}}, J_{\mathcal{A}}\right)$.

Proof. Let $G: \tilde{M} \rightarrow \mathbb{R} \times S^{2 n-1}$ be a diffeomorphism defined by $G\left(\varphi_{t} w\right)=(t, \operatorname{dev}(w))$. Note that $\operatorname{Pr} \circ G=\operatorname{dev} \circ \pi$ on $\tilde{M}$. As every element of $\mathcal{C}_{\mathcal{H}}(\mathbb{R})$ is described as $\varphi_{s} \cdot q(\alpha)$ from Remark 2.1, define a homomorphism $\Psi: \mathcal{C}_{\mathcal{H}}(\mathbb{R}) \rightarrow \mathbb{R} \times \operatorname{PSH}\left(S^{2 n-1}, \eta_{\mathcal{A}}, J_{0}\right)$ by setting

$$
\Psi\left(\varphi_{s} \cdot q(\alpha)\right)=(s, \mu(\alpha))
$$

Recall that the action $q(\alpha)\left(\varphi_{t} w\right)=\varphi_{t} \alpha w$ from (2.21). Then,

$$
\begin{aligned}
& G\left(\varphi_{s} \cdot q(\alpha)\left(\varphi_{t} w\right)\right)=G\left(\varphi_{s+t} \cdot \alpha w\right)=(s+t, \operatorname{dev}(\alpha w)) \\
& =(s+t, \mu(\alpha) \operatorname{dev}(w))=(s, \mu(\alpha))(t, \operatorname{dev}(w))=\Psi\left(\varphi_{s} \cdot q(\alpha)\right) G\left(\varphi_{t} w\right) .
\end{aligned}
$$

Hence, $(\Psi, G):\left(\mathcal{C}_{\mathcal{H}}(\mathbb{R}), \tilde{M}\right) \rightarrow\left(\mathbb{R} \times \operatorname{PSH}\left(S^{2 n-1}, \eta_{\mathcal{A}}, J_{0}\right), \mathbb{R} \times S^{2 n-1}\right)$ is equivariantly diffeomorphic. Next, since $G^{*} t=t$ for the $t$-coordinate of $\mathbb{R} \times S^{2 n-1}$ and $\operatorname{dev}^{*} \eta_{\mathcal{A}}=\eta$ from (4.10), it follows that:

$$
G^{*} \Omega_{\mathcal{A}}=G^{*} d\left(e^{t} \cdot \operatorname{Pr}^{*} \eta_{\mathcal{A}}\right)=d\left(e^{G^{*} t} \cdot G^{*} \operatorname{Pr}^{*} \eta_{\mathcal{A}}\right)=d\left(e^{t} \cdot \pi^{*} \eta\right)=\Omega .
$$

By definition, $G_{*} \xi=\frac{d}{d t}$. Moreover, when $x=\varphi_{s} w$,

$$
G\left(\psi_{t}(x)\right)=G\left(\varphi_{s} \psi_{t} w\right)=G\left(\varphi_{s} i \psi_{t}^{\prime} w\right)=\left(s, \operatorname{dev}\left(\psi_{t}^{\prime} w\right)\right)=\left(s, \mu\left(\psi_{t}^{\prime}\right) \operatorname{dev}(w)\right) .
$$

Using (2.7) and (4.7),

$$
G_{*}\left(-J \xi_{x}\right)=\left.\frac{d G \psi_{t}}{d t}(x)\right|_{t=0}=\mathcal{A}_{G x}=-J_{\mathcal{A}}\left(\frac{d}{d t}\right)_{G x} .
$$


Thus $G_{*}(J \xi)=J_{\mathcal{A}} G_{*} \xi$. As $G^{*} \Omega_{\mathcal{A}}=\Omega$ from (4.14), $G$ maps $\{\xi, J \xi\}^{\perp}$ onto $\left\{\frac{d}{d t}, \mathcal{A}\right\}^{\perp}$. Consider the commutative diagram:

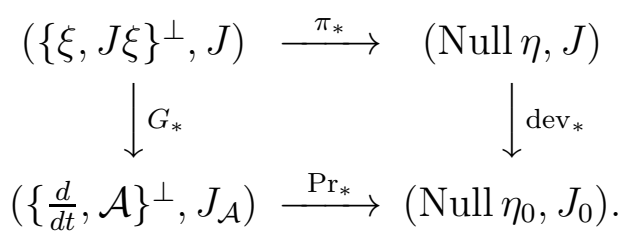

Here note that $J_{\mathcal{A}}=J_{0}$ on $\operatorname{Null} \eta_{\mathcal{A}}=\operatorname{Null} \eta_{0}$. For $X \in\{\xi, J \xi\}^{\perp}$,

$$
\operatorname{Pr}_{*} G_{*} J(X)=\operatorname{dev}_{*}\left(J \pi_{*} X\right)=J_{0} \operatorname{dev}_{*} \pi_{*}(X)=J_{\mathcal{A}} \operatorname{Pr}_{*} G_{*}(X)=\operatorname{Pr}_{*} J_{\mathcal{A}} G_{*}(X),
$$

thus, $G_{*} J(X)=J_{\mathcal{A}} G_{*}(X)$. Hence, $G$ is $\left(J, J_{\mathcal{A}}\right)$-biholomorphic. Moreover, as $G^{*} \tilde{\omega}_{\mathcal{A}}=G^{*}\left(2 e^{-t} \Omega_{\mathcal{A}}\right)=2 e^{-t} \Omega=\bar{\Theta}$ and $\bar{g}(X, Y)=\bar{\Theta}(J X, Y)$, we obtain that $G^{*} \tilde{g}_{\mathcal{A}}=\bar{g}$. Therefore, $(\Psi, G)$ induces a holomorphic isometry from $(M, \hat{g}, J)$ onto $\left(\mathbb{R} \times S^{2 n-1} / \Psi\left(\pi_{1}(M)\right), \hat{g}_{\mathcal{A}}, \hat{J}_{\mathcal{A}}\right)$.

4.3. The Hopf manifold $\mathbb{R} \times \mathbf{S}^{\mathbf{2 n}-\mathbf{1}} / \Psi\left(\pi_{\mathbf{1}}(\mathbf{M})\right)$. We prove that $\mathbb{R} \times S^{2 n-1} / \Psi\left(\pi_{1}(M)\right)$ is a primary Hopf manifold $M_{\Lambda}$ for some $\Lambda$ obtained in $\S 3$. Each element of $\pi_{1}(M)$ is of the form $\gamma=\varphi_{s} \cdot q(\alpha)$ for some $s \in \mathbb{R}$ where $\nu(\gamma)=\alpha \in Q=\nu\left(\pi_{1}(M)\right)$. By definition of $\Psi, \Psi(\gamma)=(s, \mu(\alpha))$. We show that $\Psi\left(\pi_{1}(M)\right)$ has no torsion element. For this, if $\Psi(\gamma)$ is of finite order (say, $\ell$ ), then $1=(0,1)=\Psi\left(\gamma^{\ell}\right)=\left(\ell s, \mu\left(\alpha^{\ell}\right)\right)$. Then, $s=0$ so that $\Psi(\gamma)=(0, \mu(\alpha))$. On the other hand, recall from $(4.5)$ that $\mu(Q) \subset \mathrm{U}(n-1)$ up to conjugacy, and so $\mu(Q)$ has a fixed point $w_{0} \in S^{2 n-1}$. Since $\Psi\left(\pi_{1}(M)\right)$ acts freely on $\mathbb{R} \times S^{2 n-1}$, while $\Psi(\gamma)\left(t, w_{0}\right)=\left(t, \mu(\alpha) w_{0}\right)=\left(t, w_{0}\right)$, it follows that $\Psi(\gamma)=1$. Moreover, if $\gamma_{1}=\varphi_{s_{1}} \cdot q\left(\alpha_{1}\right), \gamma_{2}=\varphi_{s_{2}} \cdot q\left(\alpha_{2}\right)$, then $\Psi\left(\left[\gamma_{1}, \gamma_{2}\right]\right)=\left(0, \mu\left(\left[\alpha_{1}, \alpha_{2}\right]\right)\right.$. By the same reason, $\Psi\left(\left[\pi_{1}(M), \pi_{1}(M)\right]\right)=\{1\}$. Hence, $\pi_{1}(M)$ is a finitely generated torsionfree abelian group. If we recall from $(2.24)$ that $1 \rightarrow \mathbb{Z} \rightarrow \pi_{1}(M) \stackrel{\nu}{\longrightarrow} Q \rightarrow 1$ is the central group extension where $Q$ is finite, then $\pi_{1}(M)$ itself is an infinite cyclic group. Since $\Psi\left(\pi_{1}(M)\right) \subset \mathbb{R} \times \operatorname{PSH}\left(S^{2 n-1}, \eta_{\mathcal{A}}, J_{0}\right)$ and the projection maps $\Psi\left(\pi_{1}(M)\right)$ onto $\mu(Q)$ in $\operatorname{PSH}\left(S^{2 n-1}, \eta_{\mathcal{A}}, J_{0}\right), \mu(Q)$ is a finite cyclic group. As $\operatorname{PSH}\left(S^{2 n-1}, \eta_{\mathcal{A}}, J_{0}\right)$ has the maximal torus $T^{n}$ (cf. (3.4)), we obtain that $\Psi\left(\pi_{1}(M)\right) \subset \mathbb{R} \times T^{n}$ up to conjugacy. A generator of $\Psi\left(\pi_{1}(M)\right)$ is described as $\left(s,\left(c_{1}, \cdots, c_{n}\right)\right) \in \mathbb{R} \times T^{n}$. Noting (4.9), let $\lambda_{j}=e^{-a_{j} s} c_{j}$ and $\Lambda=\left(\lambda_{1}, \cdots, \lambda_{n}\right)$. By Theorem 3.1 and the remark below, $\mathbb{R} \times S^{2 n-1} / \Psi\left(\pi_{1}(M)\right)$ is a primary Hopf manifold $M_{\Lambda}$ of type $\Lambda$. This finishes the proof of Theorem $\mathrm{C}$ in the Introduction.

\section{REFERENCES}

[1] F.A. Belgun, On the metric structure of non-Kähler complex surfaces, Math. Ann., 317 (2000), 1-40.

[2] D.E. Blair, Contact manifolds in Riemannian geometry, L.N.M. 509, Springer Verlag 1976.

[3] S.S. Chern and L. Greenberg, Hyperbolic Spaces, in Contribution to Analysis (A Collection of papers Dedicated to Lipman Bers, eds. L. Ahlfors and others), Academic Press, New York and London, 49-87, 1974. 
[4] P. Conner and F. Raymond, Injective operation of the toral groups, Topology, 10 (1971), 283-296.

[5] S. Dragomir and L. Ornea, Locally conformal Kähler geometry, Progress in Math. 155, Birkhäuser 1998.

[6] P. Gauduchon and L. Ornea, Locally conformally Kähler metrics on Hopf surfaces, Annales de l'Inst. Fourier, 48 (1998), 1107-1127.

[7] W. Goldman, Complex hyperbolic geometry, Oxford Mathematical Monographs, Oxford Univ. Press, 1999.

[8] Y. Kamishima, Geometric flows on compact manifolds and global rigidity, Topology, 35 (1996), 439450.

[9] Y. Kamishima, Holomorphic torus actions on compact locally conformal Kähler manifolds, Compositio Math., 124 (2000), 341-349.

[10] S. Kobayashi, Transformation groups in differential geometry, Ergebnisse der Math. 70, Springer Verlag, 1972.

[11] S. Kobayashi and K. Nomizu, Foundationsv of differential geometry II, Interscience Publisheres, New York, 1969.

[12] R. Kulkarni, On the principle of uniformization, J. Diff. Geom., 13 (1978), 109-138.

[13] J. Lelong-Ferrand, Transformations conformes et quasi conformes des variétés riemanniennes compactes, Acad. Roy. Belgique Sci. Mem. Coll., 8 (1971), 1-44.

[14] M. Obata, The conjectures on conformal transformations of Riemannian manifolds, J. Diff. Geom., 6 (1971), 247-258.

[15] M. S. Raghunathan, Discrete subgroups of Lie groups, Springer Verlag, 1972.

[16] F. Tricerri, Some examples of locally conformal Kähler manifolds, Rend. Sem. Mat. Univ. Politecn. Torino 40 (1982), 81-92.

[17] I. Vaisman, Locally conformal Kähler manifolds with parallel Lee form, Rend. Mat. 12 (1979), 263-284.

[18] I. Vaisman, Generalized Hopf manifolds, Geometriae Dedicata, 13(1982), 231-255.

[19] S. M. Webster, On the transformation group of a real hypersurface, Trans. Amer. Math. Soc., 231 (1977), 179-190.

[20] S. M. Webster, Pseudohermitian geometry of a real hypersurface, J. Diff. Geom., 13 (1978), 25-41.

Department of Mathematics, Tokyo Metropolitan University,

Minami-Ohsawa 1-1, Hachioji, Tokyo 192-0397, Japan

E-mail address: kami@comp.metro-u.ac.jp

University of Bucharest, Faculty of Mathematics

14 ACAdemiei str., 70109 Bucharest, Romania

E-mail address: lornea@imar.ro 